\title{
Günter Burkart
}

\section{Positionen und Perspektiven. Zum Stand der Theoriebildung in der Familiensoziologie}

\begin{abstract}
Zusammenfassung
Der Beitrag versucht, den Stand der familiensoziologischen Theoriebildung zu bestimmen. Dazu werden zunächst die wichtigsten theoretischen Ansätze benannt, die für die Familienforschung der letzten Dekaden in Deutschland relevant waren, gefolgt von einer Darstellung der wichtigsten theoretischen Grundprobleme und Diskussionsfelder, zunächst das Außenverhältnis der Familie betreffend, dann die Binnenstruktur. Ein Ergebnis ist, dass sich eine Reihe von Forschungsfeldern aus der Familiensoziolgie ausgelagert haben, so dass es nicht nur eine Reihe von Defiziten zu vermelden gibt, sondern dass es auch zunehmend schwierig erscheint, eine einheitliche Theorie der Familie zu konstruieren. Dies wäre aber gerade angesichts der Herausforderung durch die Erfolge der BioWissenschaften dringend notwendig.
\end{abstract}

Schlagworte: Familiensoziologie, Familientheorie, Stand der Forschung

\begin{abstract}
This paper tries to ascertain the state of the art in family theory and to identify the discussions and topics which contributed to progress in the field. For this purpose, the most influential approaches in German family sociology are discussed, followed by two sections focusing on the relationship between family and society, and the internal structure of the family, respectively. One result is that the problems of theoretical integration are increasing because some of the research fields that were formerly part of family sociology now have abandoned the field. An integrated theory of the family, however, seems to be an important issue for the future when regarding the challenge of family research by the success of biology and life-sciences.
\end{abstract}

Key words: family theory, family sociology, state of the art

\section{Einleitung}

Versteht man Familienforschung in einem sehr weiten Sinn, also einschließlich theoretischer Forschung, und Familiensoziologie als ein auch theoretisches Unternehmen, dann lässt sich feststellen, dass die Anteile von theoretischer und empirischer Forschung deutlich zugunsten letzterer verteilt sind. „Rein theoretische“ Anstren- 
gungen sind selten geworden in der Familiensoziologie, die mit ihrer empirischen Forschung ja auch wesentliche Impulse für die Praxis, für Familienpolitik und Familienberatung und den öffentlichen Diskurs liefert. Theorie ist in Praxis und Politik weniger gefragt. Sie gilt außerdem, je mehr Datensätze verfügbar sind, zunehmend als fragwürdige Spekulation. Es gibt aber durchaus eine Fülle von Versuchen, empirische Forschungen theoretisch zu reflektieren oder einzelne Studien in einen größeren theoretischen Kontext zu stellen. Insofern ist die Familienforschung sicher nicht theorielos.

Theorie-Anstrengungen können sich entweder auf die Binnenstruktur der Familie konzentrieren oder diese im Rahmen soziologischer Zeitdiagnosen und Gesellschaftsanalysen in ihren Bezügen zu gesellschaftlichen Feldern behandeln. In den letzten Jahren dominierte eher das Zweitgenannte - insbesondere die Individualisierungsdiskussion hat stark auf die Familienforschung eingewirkt. Dennoch besteht eine gewisse Gefahr, dass sich diese von der allgemeinen soziologischen TheorieEntwicklung abkoppelt. Das war einmal anders: In der Nachkriegssoziologie galt Familiensoziologie noch als wichtiger Impulsgeber für die soziologische Theorie. ${ }^{1}$ Darüber hinaus besteht die Tendenz einer internen Ausdifferenzierung in isolierte Forschungsfelder, die nicht mehr theoretisch verbunden sind (z.B. Familiendemographie, Kindheit, Jugend, Alter, Geschlechterbeziehungen, Paarbeziehungen, usw.). Damit ist schon die grundsätzliche Schwierigkeit eines solchen Übersichtsartikels angesprochen. Nicht nur, dass es für einen Einzelnen schwierig ist, den Überblick zu behalten und in seinem Urteil ausgewogen zu sein. ${ }^{2}$ Gravierender scheint mir, dass die theoretischen Anstrengungen in diesen separierten Feldern immer weniger kompatibel sind, weil sie an verschiedene Diskurse in anderen Disziplinen angebunden sind und deshalb entsprechend unterschiedlich sind, was ihre Theoriesprache anbelangt. So wäre es zum Beispiel schwierig, zwischen Familiendemographen und Ethnomethodologen einen Diskurs zu vermitteln. Außerdem sind die Grenzen des Forschungsfeldes schwer zu ziehen, weil Familie und private Lebensformen in vielen Forschungsfeldern mitberücksichtigt werden. In gewisser Weise hat auch Familie wie Geschlecht oder Lebenslauf - mehr den Charakter einer omnirelevanten Perspektive als den eines sachlich klar abgrenzbaren Gegenstandsbereichs.

1 Vgl. dazu Schmidt (2002: 384). Zur Diagnose eines „Theorie-Defizits“ vgl. etwa Vaskovics/Garhammer (1995: 2f.); Lauterbach (2003); Matthias-Bleck (2002); Schmidt (2002). Auch ein Blick auf die Tagungsthemen der Sektion Familiensoziologie (im Rahmen der Deutschen Gesellschaft für Soziologie) zeigt, dass dort überwiegend empirische und sachbezogene Probleme behandelt wurden. Eine „Theorie-Tagung“ wurde zuletzt 1998 durchgeführt, allerdings auch dort mit einer Sachorientierung (entlang des Begriffs der Solidarität; vgl. Huinink/Strohmeier/Wagner 2001).

2 Es versteht sich von selbst, dass der Autor dieses Berichts - bei allem Bemühen um Ausgewogenheit - Präferenzen hat, die einer aus Raumgründen ohnehin unvermeidlichen Selektivität des Dargestellten eine zusätzliche Einseitigkeit verleihen können. Das gilt auch für die Literaturnachweise, die selbstverständlich alles andere als vollständig sein können. Ich danke den KollegInnen, die während oder nach der Bamberger Tagung mit Hinweisen und Kritik geholfen haben, insbesondere Angelika Tölke sowie Johannes Huinink und Michael Wagner. 
„Familiensoziologie“ ist der in Deutschland eingebürgerte Terminus. Wegen der vielfältigen Veränderungen der letzten Jahrzehnte, die zu einem Bedeutungsverlust der klassischen Familie oder der Familie überhaupt geführt zu haben scheinen, zweifeln einige Autoren die Berechtigung dieser Bezeichnung an (etwa, weil die Subsumption des Paares unter die Ehe, und der Ehe unter die Familie, nicht mehr akzeptiert wird). Doch über die Alternative gibt es keine Einigkeit, und man kann dies auch als Symptom einer theoretischen Unsicherheit betrachten. Ist Familie eine Institution mit bestimmten Normen und Funktionen? Ist sie ein System (oder Teil eines anderen Systems oder besteht sie aus Teilsystemen)? Ist sie eine von mehreren Lebensformen oder sozialen Beziehungstypen, eine neben anderen oder doch noch eine hervorgehobene Form?

Zunächst werden die wichtigsten theoretischen Ansätze benannt, die für die Familienforschung der letzten Dekaden in Deutschland relevant waren (1.). Es folgt eine Übersicht zum Stand der Diskussion in den Forschungsfeldern und Themengebieten, die das Außenverhältnis der Familie betreffen (2.). Eine Darstellung des Problemstandes zur Binnenstruktur schließt sich an (3.), bevor am Ende ein kurzer Blick in die Zukunft geworfen wird (4.).

\section{Soziologische Theorie und Familie}

\subsection{Theorien in der deutschen Familiensoziologie}

Es sind im Wesentlichen drei Theorie-Richtungen, die in den letzten 20 Jahren der deutschsprachigen Familienforschung deutliche Impulse geben konnten: die Individualisierungstheorie, die Theorie funktionaler Differenzierung und die RationalChoice-Theorie.

a) Individualisierungstheorie. Den stärksten Einfluss auf die allgemeine familiensoziologische Diskussion in Deutschland hatte in den letzten zwei Dekaden die Individualisierungsthese, die neben der Familien- vor allem auch in der Jugend- und Kindheitsforschung, aber auch in Teilen der Geschlechterforschung spürbar wirksam war. Einige Publikationen Mitte der 1980er Jahre leiteten diesen Trend ein (Beck, BeckGernsheim, Kohli u.a.). ${ }^{3}$ Seither gab es unzählige Publikationen, in denen immer wieder die Individualisierung und Pluralisierung der Lebensformen konstatiert wurde. Die Individualisierungsthese wurde manchmal als Krisen- und Verfallsdiagnose der Familie vorgetragen, häufiger aber als Pluralisierungs- und Entstrukturierungsthese (Ausfächerung von Familien- und Lebensformen und ein gewisses Beliebigwerden dominanter Formen). Die Grundaussagen der Individualisierungsthese - Autonomisierung der Familie vom Verwandtschaftssystem und der Individuen von der Familie - gehören seit Durkheim und Simmel zum common sense der Familienso-

3 Einige von ihnen wurden später in einem Sammelband zusammengefasst (Beck/BeckGernsheim 1994). 
ziologie (Singly 1994). Einer der zahlreichen Streitpunkte, die hier insgesamt nicht mehr genannt werden müssen, war deshalb die Frage nach den Spezifika eines neuen Individualisierungsschubes.

b) Differenzierungstheorie. Auch die Theorie funktionaler Differenzierung, im Anschluss an die Klassiker, vor allem aber an Parsons und Luhmann, hat starken Einfluss auf die theoretische Familiensoziologie ausgeübt, wenn auch weniger spektakulär als im Fall der Individualisierungsthese. Zu nennen sind hier neben Nave-Herz vor allem die Arbeiten von Bielefelder Autoren, im Anschluss an Luhmann. ${ }^{4}$ Mit der These von der De-Institutionalisierung (Tyrell 1988) näherte sich die Differenzierungstheorie der Individualisierungsthese, und selbst Luhmann wird inzwischen als Theoretiker der Individualisierung interpretiert (Willems 1999; Schroer 2000): Funktionale Differenzierung führt zu einem zunehmenden Bedarf an Individualität und Selbstthematisierung. Diskutiert wurde auch die Ausdifferenzierung des Intimsystems bzw. die These der Entkopplung von Ehe und Elternschaft. In langfristiger Perspektive ist wohl unbestritten, dass es zur Ausdifferenzierung eines Intimsystems (Privatsphäre, Familie) gekommen ist. Weniger klar ist, wie heute das Verhältnis verschiedener Teilbereiche -Verwandtschaft, Familie, Paarbeziehungen, Intimität, Privatheit - zueinander verstanden werden soll.

c) Rational-Choice-Theorien. ${ }^{5}$ Im Anschluss an die ökonomischen Theoretiker früherer Generationen (Th. Schultz, G. Becker, H. Leibenstein), haben deutsche Rational-Choice-Theoretiker ihre allgemeinen Überlegungen auch auf die Familie angewandt (Hill/Kopp 1995; Esser 1999) und einen spürbaren Einfluss auf die soziologische Familienforschung gewonnen. Die Rational-Choice-Theorie kommt vielen Empirikern entgegen, weil sie am Individuum ansetzt und Ergebnisse, die aus Befragungen von Individuen gewonnen wurden, mit adhoc-Erklärungen plausibilisieren kann. Sie ist als Handlungstheorie konzipiert, die offen ist für die Berücksichtigung von die Rationalität einschränkenden Strukturbedingungen. ${ }^{6}$ In welcher Weise verhindern bestimmte Bedingungen (constraints), dass Menschen rational handeln können? Wichtig sind auch Überlegungen zur Mikrofundierung von Makrostrukturen (Esser 2001), zu den nichtintendierten Handlungsfolgen, wie sie sich etwa in Veränderungen der Fertilitäts- oder Scheidungsraten niederschlagen. Allerdings wird immer

4 Meyer (1992); Herlth et al. (1994); F.-X. Kaufmann (1994). Zu Luhmanns Familientheorie vgl. auch Burkart (2005). Manchmal ist hier auch synonym von „Modernisierungstheorie“ die Rede (Nave-Herz 1999).

5 Die Bezeichnung umfasst hier verschiedene Ansätze wie Haushaltsökonomie, Mikroökonomie der Familie, Austausch-, Ressourcen- oder Entscheidungstheorien, sofern sie in der Tradition von Theorien des ökonomischen Austausches stehen (Nutzenorientierung, quantifizierbare Tauschrelationen). Dagegen sind Theorien des symbolischen Tauschs, im Anschluss an Simmel, Mauss (Gabentausch) und Goffman hier natürlich nicht gemeint (vgl. dazu etwa Hochschild 1989). In der englischsprachigen Familienliteratur findet man den Ausdruck rational choice immer noch selten, eher ist von exchange theory die Rede.

6 Und auch offen für Kooperationen mit der Psychologie, die ansonsten in der Familiensoziologie relativ selten geworden sind - wie der regelmäßige Blick in diese Zeitschrift belegt, in der zwar beide Richtungen vertreten sind, aber selten aufeinander Bezug nehmen. 
wieder die enge Bindung an das ökonomische Modell der rationalen Wahl kritisiert, das den soziologischen Wert der Theorie in Frage stellt. Umstritten ist auch, welchen Status die theoretischen Rational-Choice-Aussagen haben: Sind es lediglich Als-obModell-Aussagen auf der Mikro-Ebene? Handelt es sich um die Behauptung, im empirischen Normalfall liege dem Handeln immer eine rationale Wahl nach einer Kosten-Nutzen-Kalkulation zugrunde? Oder handelt es sich um eine normative Theorie (im Sinne einer Vernunftmoral)? Ein Großteil der kritischen Diskussion kämpft mit entsprechenden Missverständnissen. Kritik gibt es auch an der Nichtberücksichtigung von Erkenntnissen der Geschlechterforschung (Katz 1997; Schulz/Blossfeld 2006).

d) Andere Theorien. Welchen Einfluss haben andere soziologische Theorien auf die Familienforschung? Zwar wird der Strukturfunktionalismus Parsons'scher Prägung, der die deutsche Familientheorie der 1950er Jahre dominierte, noch in vielen Übersichts- und Lehrbüchern als einer von fünf oder sechs der wichtigsten Ansätze genannt, spielt jedoch seit längerem keine führende Rolle mehr, zumindest nicht im Sinne einer expliziten Bezugnahme. Gleichwohl müssen sich die an Pluralisierung oder Auflösung orientierten Thesen, denen zufolge es ,die Familie“ oder eine feste Struktur der Familie nicht mehr gibt, weiterhin am klassischen Modell der Struktur der Kernfamilie - die sich als Kreuztabelle zweiter dichotomer Variablen, Geschlecht und Generation, darstellen lässt - orientieren. Auch die Frage nach Leistungen und Funktionen der Familie ist nicht obsolet geworden. Was aber mit der Vernachlässigung von Parsons' Ansatz verloren gegangen ist, ist die Verknüpfung von drei Ebenen: Makro-Strukturen (Kultur, Ökonomie, Gesellschaft), Interaktionssystem Familie und Persönlichkeitsstruktur. Parsons hatte ja seine Familientheorie auch auf die Psychoanalyse aufgebaut (was viele heute gar nicht mehr wissen und was auch nicht zu dem Bild des konservativen Familienideologen passt, das die meisten Studierenden heute von Parsons vermittelt bekommen). ${ }^{7}$

Der Symbolische Interaktionismus, der eine Zeitlang stark die amerikanische Familienforschung beeinflusste, war in Deutschland in der Familienforschung weniger präsent. Neuerdings gibt es allerdings aus interaktionistischen Richtungen (in einem weiten Sinn) Bestrebungen, das Feld der Familiensoziologie auszuweiten und die Verengungen, die mit dem (teilweise als überholt bezeichneten) Familienbegriff verbunden sind, aufzubrechen. Aus der Familiensoziologie sollte, so die Forderung, eine Soziologie der Intimbeziehungen, der persönlichen Beziehungen (Lenz 2003, 2005) oder der Privatheit (Schneider 2002) werden - oder eine „Sozialpsychologie der Ehe“, wie Bertram (2002) kritisch anmerkt. ${ }^{8}$ Es spricht sicher manches dafür,

7 Vereinzelt finden sich Bezugnahmen auf andere Klassiker, zum Beispiel Durkheim (Wagner 2001) oder Simmel (Tyrell 2001). Aus dem Umfeld der strukturtheoretischen Soziologie stammt der Versuch von Allert (1998), die „Unverwüstlichkeit“" von Familie gegenüber den behaupteten Auflösungstendenzen nachzuweisen.

8 Vgl. dazu die Theorie-Debatten um den Kern (und die Bezeichnung) des Forschungsgebietes in einer Ausgabe des Diskussionsforum Erwägen - Wissen - Ethik (Lenz 2003 und Diskutanten) und in Soziale Welt (Schneider 2002, mit Kritiken von Bertram, Burkart und Matthias-Bleck). 
Ehe und Familie nicht mehr in erster Linie als Institution oder als System zu betrachten, sondern als eine Form von persönlichen Beziehungen zu begreifen, denn zum Beispiel zeigt ja die Scheidungsforschung, dass die höheren Scheidungsraten zu einem erheblichen Teil auf gestiegene Ansprüche zurückgeführt werden müssen - also die Qualität der persönlichen Beziehung stärker im Vordergrund steht als früher. Aber eine Reduzierung der Familie auf persönliche Beziehungen oder Interaktionsformen wäre für die Soziologie doch unzureichend.

Wie in der gesamten Soziologie, hat auch im Bereich der Familienforschung der Marxismus in wenigen Jahren in der zweiten Hälfte der 1970er Jahre fast jeden Einfluss verloren. Das könnte sich vielleicht wieder ändern, in der Soziologie scheint der Begriff des Kapitalismus wieder salonfähig zu werden. Für Hochschild (1997, 2003) ist es der Kapitalismus, der das Verhältnis von Familie und Arbeit und deren emotionale Besetzung nachhaltig ändert. ${ }^{9}$ In Lehr- und Übersichtsbüchern findet man manchmal noch Bezeichnungen wie „Konfliktansatz“ oder „kritische“ Familienforschung.

Schließlich ist keine Frage, dass der Feminismus und die Geschlechterforschung wichtige Impulse für die Familientheorie geliefert haben, wenn auch deren theoretisches Interesse nicht primär auf die Familie gerichtet war und feministische Studien zur Familie relativ selten sind. Von einer feministischen Familienforschung kann man deshalb wohl nicht sprechen - und die Phase feministischer Familienkritik ist möglicherweise schon historisch. ${ }^{10}$ In den USA gibt es allerdings Ansätze für eine postmodern-feministische Familientheorie (Baber/Allen 1992; Osmond/Thorne 1993), die in der Tradition der feministisch-konstruktivistischen Kritik am bürgerlich-männlichen Familien- und Geschlechtermodell steht.

\subsection{Theorie-Probleme}

Probleme der Familientheorie lassen sich an drei Punkten aufzeigen: dem Verhältnis zur Empirie, dem Familienbegriff (der Abgrenzung und Eingrenzung des Gegenstandsbereichs) und dem Verhältnis zur allgemeinen soziologischen Theorie.

Theorie und Empirie. Die genannten Theorie-Richtungen haben zwar die empirische Forschung angeregt, sind aber nicht so konzipiert, dass sie unmittelbarer Überprüfung zugänglich wären. Über die empirische Fundierung der Individualisierungsthese wurde bekanntlich viel gestritten (Beck/Beck-Gernsheim 1993, Friedrichs 1998). Auch die Theorie der funktionalen Differenzierung gibt keine klaren Kriterien etwa für die empirische Abgrenzung zwischen verschiedenen familialen Teilsystemen. Die Rational-Choice-Theorie scheint auf den ersten Blick, wie schon angedeutet, forschungsnäher. Aber Kosten-Nutzen-Kalkulationen und Entscheidungspro-

9 Für einige Autoren (z.B. Boltanski/Chiapello 2003) profitiert der Kapitalismus heute von jenen Tendenzen, die der Familie geschadet haben, nämlich den Trend zu Individualismus und Selbstverwirklichung.

10 Vgl. etwa das Themenheft der Feministischen Studien zu Kinderlosigkeit, dessen Herausgeberinnen auf Distanz zur früheren feministischen Familienkritik gehen (Benninghaus 2005). 
zesse werden selten direkt empirisch untersucht, häufig bleibt die rationale Wahl eine bloße Annahme (Blossfeld/Müller 1996; Burkart 2002). Es ist deshalb nicht überraschend, dass sich die empirische Familienforschung immer mehr von der Theorie verselbständigt hat. Es scheint fast so, als ob die Theorie-Anbindung der Forschung schwächer wird, je ausgefeilter die methodischen Instrumente werden und je klarer die Selbstverständlichkeit wird, sich auf bestimmte Datensätze zu beziehen.

Arbeit am Familienbegriff. Diskussionen des Familienbegriffs sind ein gewisser Indikator für theoretische Entwicklungen. Allerdings ist nicht zu übersehen, dass damit meist auch normative Positionierungen verbunden sind. So ist zum Beispiel in den letzten Jahren deutlich geworden, dass Autoren häufiger darauf verzichten, a) die Rechtsform Ehe, b) die Anwesenheit zweier Eltern, c) die Anwesenheit eines heterosexuellen Elternpaares als Grundelement der Definition von „Familie“ zu betrachten. Das sind nicht immer theoretisch oder empirisch begründete Setzungen, sondern manchmal eher implizite politische Stellungnahmen: a) für nichteheliche Lebensgemeinschaften, b) für Ein-Eltern-Familien (Alleinerziehende), c) für homosexuelle Elternpaare - für diese drei Gruppen wird damit „Familien“-Status reklamiert. Die familiensoziologische Theorie trägt hier zwar den gewandelten normativen Haltungen - dem „Diskurs“ - in der Kultur Rechnung, muss sich aber fragen lassen, ob damit die Struktur schon ausreichend beschrieben ist. Klärungsbedürftig wäre beispielsweise die Frage nach der Vaterposition, die aufgrund empirischer Befunde als teilweise verzichtbar gilt oder als austauschbar. „Familie“ wäre dann im Kern reduzierbar auf die Mutter-Kind-Dyade (,Neue Matrilinearität“).

Allgemeine soziologische Theorie und Familienforschung. Inwiefern und wie werden in den allgemein-soziologischen Entwürfen oder Ausarbeitungen der letzten Jahre und Jahrzehnte familienrelevante Fragestellungen erörtert? Und umgekehrt: Inwiefern haben die Familienentwicklung und die Familienforschung die allgemeine Theorie-Produktion inspiriert? Tatsächlich wurden die genannten drei TheorieAnsätze (Individualisierungsthese, Rational-Choice und die Theorie funktionaler Differenzierung) nicht als Theorie der Familie entworfen, thematisieren aber auch die Familie. Dies gilt für andere soziologische Theorien nur noch bedingt; dort herrscht weitgehend Fehlanzeige, wenn man nach Bezügen zur Familie sucht. In der Zeit, als die Familienforschung vom Strukturfunktionalismus geprägt war, interessierte sich die allgemeine soziologische Theorie noch für die Familie als Gegenstand, an dem Theorie-Entwicklung stattfinden kann. Schelsky oder König hatten noch Wesentliches zur Familie zu sagen, im Unterschied zu später führenden soziologischen Theoretikern wie Habermas oder Luhmann. Auch Bourdieu hat kaum etwas zur modernen Familie geschrieben. ${ }^{11}$ Ebenso finden wir in den zahlreichen so-

11 Die im Umfeld von Habermas geführte Debatte über das Verhältnis von Zivilgesellschaft und Familie wurde in der deutschen Familiensoziologie kaum zur Kenntnis genommen (Ostner 1997). - Für Bourdieu ist die Familie zunächst der Ort, an dem der Klassenhabitus erworben wird und die Institution, die für die Reproduktion der Klassenstruktur entscheidend ist. Auch in den Passagen zur Familie in La domination masculine (Bourdieu 1998) wird die Familie als Ort dargestellt, in dem nach wie vor - trotz beruflicher Emanzipation - darauf geachtet wird, über die Heirat das soziale und symbolische Kapital der Familie zu bewahren oder zu mehren. 
ziologische Gegenwartsdiagnosen nur wenige Bezüge auf Familie. In einer Überblicksdarstellung verschiedener Zeitdiagnosen (Schimank/Volkmann 2000) kommt „Familie“ nur im Artikel über den Kommunitarismus in dessen konservativer Spielart vor, wo es um das moralisch-politische Argument geht, die Familie müsse gestärkt werden, weil dort die Werte der guten Gesellschaft am besten aufgehoben seien. In den übrigen Zeitdiagnosen spielt die Familie kaum eine Rolle oder nur vermittelt über die Veränderungen des Geschlechterverhältnisses (wie bei Beck und Giddens), wenn es um die Auswirkungen der Modernisierung auf die Geschlechtsrollen in der Familie geht.

Hinzu kommt ein Differenzierungsprozess innerhalb der Familiensoziologie, der sich seit den 1980er Jahren verstärkt hat. Institutionell zeigt sich dies besonders klar in der bereits genannten Ausdifferenzierung von Forschungsbereichen. Kindheit, Jugend, Alter(n), Geschlecht, Sozialisation, Lebenslauf und Biographie - diese Bereiche kommen in der Familiensoziologie (hier verstanden im institutionellen Sinn der Sektion innerhalb der Soziologie) kaum noch vor. Dies hat mit dazu beigetragen, dass es seit etwa Anfang der 1990er Jahre eine Dominanz familiendemographischer Fragestellungen gibt. Auch die Thesen von der Pluralisierung der Lebensformen und der Abkehr von der klassischen Normalfamilie führten zu einer Konzentration auf demographisch gut erfassbare Phänomene: Wie viele „Normal“-Familien gibt es noch, wie viele Singles, wie viele Alleinerziehende? Was sind die relevanten demographischen Variablen zur Erklärung von Veränderungen der Scheidungsquote? Mit dieser Demographisierung der Familiensoziologie wurden bestimmte Bereiche theoretisch und empirisch systematisch vernachlässigt: Sexualität, Liebe, Emotionen, Körperlichkeit, aber auch Interaktion und Kommunikation. „Merkwürdigerweise ist es der modernen Familiensoziologie gelungen, Sexualität, erotische Gefühle und Emotionen, wie Liebe und Hass, weitgehend aus ihrer Arbeit auszublenden“" (Bertram 1995).

\subsection{Theorie in der internationalen Familiensoziologie}

Dieser Punkt kann hier nur knapp skizziert werden. Eine Durchsicht englischsprachiger Handbücher und Einführungsbücher mit der Frage nach dem Anteil von "Theorie“" ergibt kein wesentlich anderes Bild als für die deutschsprachige Forschung. Ein Großteil dieser Publikationen ist deskriptiv, problem- und sachorientiert, bietet also kaum „Theorie“ im klassischen Sinn (z.B. Gelles 1995; Leira 1999; Scott et al. 2004). Deutlicher ist aber eine globale Orientierung auszumachen und ein stärkeres Interesse am Kulturvergleich. ${ }^{12}$ Auch scheint man international eher der Auffassung zu sein, dass Familie (einschließlich Lebensformen, persönliche Beziehungen, Privatheit) ein zentraler Bereich der globalisierten Welt ist: „For examining the impact of globalization and the ramifications of individualization, there is no better

12 „Families in a global world“ ist ein Hauptteil eines Sammelbandes (Scott et al. 2004) überschrieben. Von einem „Western bias“ in der Familiensoziologie ist dort die Rede; gleichwohl spiegelt der Band mit vorwiegend nordamerikanischen und britischen Autoren diese Einseitigkeit wieder. 
test-bed than the family setting" (Scott et al. 2004, xv). Ähnlich auch Giddens (1999: 51). Arlie Hochschild arbeitet an einer Zeitdiagnose, deren wichtigste Themen sind: „emotion, gender, family, capitalism, globalization“ (2003: 1). Hochschild gehört international zu den wenigen, die versuchen, den Gesamtzusammenhang zwischen Geschlechterbeziehungen, Familie und Arbeitswelt im Auge zu behalten und dabei sowohl die Bedeutung von Emotionen zu berücksichtigen als auch die Tendenzen zur Professionalisierung von Familientätigkeiten (care work) nicht zuletzt durch Globalisierung (outsourcing of care work) (Hochschild 2003).

Im Sammelband von Gelles (1995) werden als die fünf wichtigsten TheorieAnsätze genannt: Strukturfunktionalismus, Symbolischer Interaktionismus, developmental approach (Lebensphasen), Konflikt- sowie Austauschtheorien. Im Licht neuerer Arbeiten wirkt diese Liste allerdings etwas überholt, zunehmend findet sich eine Dominanz „alternativer“ Ansätze: postmodern, feministisch, postkolonial usw. Diesen Ansätzen wurde in den 1990er Jahren zunehmend eine starke Stellung in englischsprachigen Handbüchern und Sammelbänden zugewiesen (Doherty 1999; Cheal 1999). ${ }^{13}$ Postmoderne Merkmale, die auch die privaten Lebensformen kennzeichnen, sind Fragmentierung, Fluidität, Pluralismus und Diversifizierung, Fortschritts- und Rationalitätsskepsis. Die postmoderne Familie macht, wie schon Shorter (1975) bemerkte, eine Reise ins Ungewisse. ${ }^{14}$ Unsicherheit und Pluralismus von Lebensformen - hier gibt es in der Sache deutliche Überschneidungen zur Individualisierungstheorie. Unter dem Stichwort „Individualisierung“ findet man in englischsprachigen Werken häufig noch den Hinweise, dass diese Theorie vor allem in Deutschland verbreitet sei (Cheal 1999). Allerdings gab es auch in den 1980er Jahren schon US-Autoren, die ganz ähnlich argumentierten (z.B. Cherlin/Furstenberg 1989).

\section{Familie im gesellschaftlichen Kontext}

\subsection{Funktionen der Familie und Funktionswandel}

Es gibt eine ehrwürdige Tradition in der sozialwissenschaftlichen Denkgeschichte, die Familie als Gegenstruktur zur Gesellschaft anzusehen, als sicheren Hafen in ei-

13 In der zweiten Auflage des Handbook of Marriage and the Family (Sussmann/Steinmetz/ Peterson 1999) ist einer von zwei Theorie-Artikeln der postmodernen Familientheorie gewidmet (Doherty 1999) - und ist der einzige, der die neuere Theorie-Entwicklung rekapituliert. Postmodern ist häufig eine Art Sammelbegriff für feministische Studien, postcolonial studies und andere Richtungen, wo es um Minderheiten-Schutz und gegen die Hegemonie der männlich-rationalen Mainstream-Familientheorie geht. Auf der anderen Seite ist bemerkenswert, dass der Anteil von Artikeln über alternative Lebensformen (gegenüber der Erstauflage 1987) wieder zurückgegangen ist, sogar der Artikel über freiwillige Kinderlosigkeit wurde gestrichen.

14 Setting the course for the heart of the sun - so ein Pink Floyd-Titel, den Shorter (1975) als Kennzeichen der postmodernen Familie der kalifornischen Subkultur wählte. 
ner unruhigen Welt, als Hort des Privaten und der Intimität, als Rückzugs- und Schutzraum gegen die „kalten“ (rationalisierenden) Tendenzen der modernen Gesellschaft. Sie hatte aber in den letzten Jahren kaum noch Einfluss auf die Theoriebildung in der Familiensoziologie. Allerdings kommt diese Perspektive zum Teil noch in jenen Ansätzen zum Ausdruck, die in der gesellschaftlichen Modernisierung eine Bedrohung der Familie sehen. ${ }^{15}$ Andere Ansätze heben demgegenüber die große Bedeutung für die Gesellschaft hervor. So wird etwa in der Theorie der Zivilgesellschaft die Familie als wichtige intermediäre Instanz zwischen Markt und Staat betrachtet (Cohen/Arato 1992). Und im Kontext der Theorie funktionaler Differenzierung bzw. der Modernisierungstheorie wird weiterhin ganz unspektakulär nach Aufgaben, Leistungen und Funktionen der Familie für die Gesellschaft und deren Teilbereiche gefragt. ${ }^{16}$

Als Funktionen werden Leistungen oder Beiträge für andere Bereiche und für die Gesamtgesellschaft angesehen, und dabei geht es auch um Tendenzen der Erosion solcher Funktionen. Hier sind vor allem Arbeiten von F.-X. Kaufmann zu nennen (1990 bzw. 1994; vgl. auch Nave-Herz 2004: 77ff.). Auch wenn die Rede vom „Funktionsverlust“ häufig kritisiert wurde - historisch gesehen lässt sich insgesamt doch ein gesellschaftlicher Bedeutungsverlust der Familie konstatieren (und nicht nur eine Funktions-Spezialisierung). Mit dem Übergang zur Moderne verlor die Familie eine Reihe von politischen, ökonomischen und rechtlichen Funktionen. Andere blieben erhalten oder differenzierten sich weiter aus - theoretisch gibt es dazu aber wenig Neues in den letzten Jahren. Der Stand lässt sich vielleicht so zusammenfassen, dass vier Grundfunktionen unterschieden werden können: Biologische und soziale Reproduktion, Sozialisation und Statuszuweisung. Die Diskussion dreht sich vor allem um die Frage der Schwächung dieser Funktionen.

Nach wie vor gilt die biologische Reproduktion der Bevölkerung als eine zentrale Funktion der Familie, d.h. sie hat ein Privileg für die Zeugung von Kindern (anders gesagt: Wenn die Gesellschaft ihre Nachwuchsproduktion sichern und steuern will, wird sie sich zuerst mit der Frage der Absicht zur Familiengründung bei Paaren befassen). Zwar gibt es Anzeichen der Schwächung dieser familialen Funktion im Sinne einer Stärkung matrilinearer Tendenzen und einer relativen Schwächung der Konjugalität: Immer häufiger wird Mutterschaft ohne klassische Familienkonstellation konstituiert oder fortgesetzt. Aber immer noch leben etwa 80 Prozent der Kinder bei ihren beiden biologisch-sozialen Eltern. Wegen des weitgehend durchgesetzten Prinzips der „,verantworteten Elternschaft“ (Kaufmann 1994: 42) verweist die biologische Reproduktionsfunktion „heutzutage sogar enger als je zuvor - auf Familie“ (Nave-Herz 2004: 85). Ernsthaft bedroht wäre das Monopol der Familie auf die biologische Reproduktionsfunktion daher erst durch Tendenzen einer Professionalisierung der Elternschaft, d.h., wenn durch einen zu hohen Grad an Kinderlosigkeit

15 Bellah et al. (1985), Hochschild (2003). Zelizer (2005) kritisiert, dass eine solche Kolonialisierungs- oder Rationalisierungskritik die Familie ungewollt naturalisiere, als ob es dort eine Art natürlicher Wärme gäbe.

16 Die funktionalistische Perspektive wäre auch hilfreich in Bezug auf alternative Lebensformen. Allerdings wird selten präzise gefragt, ob neue Lebensformen funktionale Äquivalente für die Familie sein könnten. 
oder ein zu niedriges Geburtenniveau eine Form von sozialer Leihmutterschaft - gewissermaßen „Berufsmutterschaft“ - legitimiert würde. Dafür gibt es durchaus Anzeichen, insbesondere im Zusammenhang mit der Globalisierung (Hochschild 2003), der Vereinbarkeitsproblematik bei Akademikerpaaren und der Gen-Technologie.

Analysen zur Sozialisationsfunktion, die in der Forschung der 1970er Jahre noch im Vordergrund standen, spielen heute in der Familiensoziologie eine untergeordnete Rolle. Dabei hat ganz besonders in Deutschland die Familie praktisch das Monopol für die Kleinkind-Sozialisation - Huinink (2002) spricht von „kulturellem Familismus". Die Anforderungen an gute Erziehung sind weiter gestiegen (Nave-Herz 2004: 88 ff.; Apple 2006), das fördert allerdings auch Professionalisierungstendenzen im Sinne der Auslagerung bestimmter Sozialisationsleistungen aus der Familie, die sich überfordert sieht. Auch Elemente der sozialen Reproduktionsfunktion werden zunehmend ausgelagert, zum Beispiel Kochen, Essen oder Waschen, ebenso viele Freizeitbeschäftigungen. Dennoch gibt es Anzeichen, dass diese Funktion wichtiger geworden ist. Manche Autoren heben deshalb den Spannungsausgleich hervor, also soziale Reproduktion im Sinne von Erholung und Regeneration. ${ }^{17} \mathrm{Be}$ tont wird auch die „Versorgung" der Familienmitglieder mit affektiven Bindungen, Solidarität, Intimität und emotionaler Sicherheit in einem basalen Sinn. Aber auch hier sehen manche Autoren Gefahren der Erodierung dieser Funktion. Hochschild etwa spricht vom Auskühlen der emotionalen Wärme in der Privatsphäre durch Tendenzen der Kommerzialisierung der Intimität (Hochschild 2003), aber auch von Tendenzen der Rationalisierung: „When home becomes work“ (Hochschild 1997).

Schließlich hat die Herkunftsfamilie für die Kinder immer noch eine große Bedeutung im Sinne der sozialen Platzierung und Statuszuweisung. Dem Bildungssystem kommt zwar eine vermittelnde Funktion bei der Statuszuweisung zu (es bietet Chancen für sozialen Aufstieg), aber es scheint, dass sich von Bourdieus frühen Untersuchungen bis zu den PISA-Studien sowohl in empirischer als auch in theoretischer Hinsicht wenig geändert hat an der grundlegenden Diagnose der Reproduktion sozialer Ungleichheit über die Familien. ${ }^{18}$

Der Familie kommen also nach wie vor die eng zusammenhängenden Funktionen der biologischen Reproduktion, der Sozialisation, der Statuszuweisung und der sozialen Reproduktion (von einfacher Regeneration bis zu Selbstverwirklichung) zu. Sie hat die Aufgabe und das Monopol der Nachwuchssicherung (Geburt, Pflege und Primär-Erziehung von Kindern), der Reproduktion der Individuen in der Intimsphäre und damit letztlich auch die Funktion, Individualität herzustellen.

\subsection{Familie und gesellschaftliche Felder}

Die Familie mag, wie Parsons formulierte, „strukturell isoliert“ sein - gleichwohl steht sie in vielfältigen Verbindungen, Interdependenzen und Spannungsverhältnis-

17 Während der Marxismus dies noch kritisch bewertet hatte - die Reproduktion diene vor allem dazu, die Menschen wieder arbeits- und damit ausbeutungsfähig zu machen -, sind solche Stimmen heute nur noch selten zu hören

18 Bourdieu/Passeron (1971); Huinink (2000). 
sen zu anderen Bereichen. Man könnte sagen, dass die Familie die Basis für verschiedene gesellschaftliche Bereiche und Makrostrukturen darstellt. Indem sie ihrer biologischen Reproduktionsfunktion nachkommt, stellt sie der Gesellschaft das Personal zur Verfügung, das sie darüber hinaus - über die Erfüllung der Sozialisationsund der sozialen Reproduktionsfunktion - mit grundlegenden Kompetenzen und Werthaltungen ausstattet und dessen Leistungsfähigkeit sie immer wieder erneuert. Schließlich trägt sie dazu bei - über die Statuszuweisungsfunktion -, dieses Personal auf Statuspositionen zu verteilen.

Die Forschungen der letzten Jahre hinsichtlich der Beziehungen der Familie zu anderen gesellschaftlichen Bereichen haben sich stark auf die Bevölkerungsstruktur konzentriert. Ein Großteil der Diagnosen zum Wandel der Familie stützt sich auf Daten zur demographischen Entwicklung, insbesondere zu Fertilität und Nuptialität. Der drohende Untergang der Familie wurde eng mit dem Geburtenrückgang - zuletzt der wachsenden Kinderlosigkeit -, aber auch mit anderen demographisch feststellbaren Tendenzen in Zusammenhang gebracht: sinkende Heiratsneigung, steigende Scheidungsquoten, steigende Quoten alternativer Lebensformen, insbesondere Singles sowie kinderlose, nichtverheiratete Paare. Theorien zum Geburtenrückgang sind jedoch nur schwach verknüpft mit allgemeinen soziologischen Diagnosen. ${ }^{19}$ Es gibt den einen oder andern Versuch der Begründung einer theoretisch orientierten Bevölkerungssoziologie (Höpflinger 1997), in der allgemeinen Soziologie aber existiert der Bevölkerungsbegriff als Theorie-Begriff praktisch nicht. Theoretische Überlegungen zum Wandel der Bevölkerungsstruktur finden sich daher eher in der Ökonomie - im Anschluss an Malthus - und in der bevölkerungswissenschaftlichen Familiendemographie.

Die Aufmerksamkeit eines Großteils der Familienforschung, sofern sie auf $\mathrm{Ar}$ beits-, Berufs- oder Beschäftigungssystem bezogen ist, galt dem „Vereinbarkeitsproblem“ oder, wie es neuerdings heißt, der work-life-balance und der Frage von „Familienfreundlichkeit" von Unternehmen. Für die Theoriebildung scheint dieses Problem zunächst wenig ergiebig, da es dabei vorwiegend um die Frage von adäquaten infrastrukturellen oder politischen Maßnahmen geht, mit denen die Hoffnung verbunden wird, den Frauen und Paaren die Entscheidung für ein Kind zu erleichtern. Allerdings wurden hier in den letzten Jahren wichtige Fragen der Flexibilisierung von Arbeit und Familie und der Veränderungen der entsprechenden Zeitstrukturen aufgeworfen.

\subsection{Familie und Geschlecht}

Die Verknüpfungen von Familien- und Geschlechtertheorie gestalten sich für Mikround Makro-Ebene unterschiedlich. In diesem Abschnitt geht es um das Verhältnis von Familie und Geschlecht auf der Makro-Ebene, also um die Interrelationen zwi-

19 Die deutlichsten Annäherungen zwischen sozialwissenschaftlicher Zeitdiagnose und Theorien des Geburtenrückgangs gibt es wohl in der Theorie des „Zweiten demographischen Übergangs“" (Lesthaeghe). 
schen dem Familiensystem und dem System der „Geschlechterklassen“ (Goffman 1994: 107 ff.). Die Binnenstruktur (Geschlechterverhältnis in Paarbeziehungen und Familien) wird in einem späteren Abschnitt behandelt (3.5).

Nach wie vor ist das Verhältnis der beiden Forschungsfelder Geschlechter- und Familiensoziologie zueinander angespannt, auch wenn innerhalb der Familienforschung etwa die Arbeitsteilung zwischen den Geschlechtern (vor allem in Verbindung mit der „Vereinbarkeitsproblematik“) ein zentrales Thema geworden ist. Aber die theoretischen Diskussionen der Geschlechterforschung wurden in der Familiensoziologie nur am Rande rezipiert. Die von Goffman stammenden wichtigen Impulse und die Debatten um soziale Konstruktion von Geschlecht wurden nur selten aufgegriffen (Goffman 1994; Tyrell 1986; Burkart/Koppetsch 2001). Umgekehrt befassen sich die feministische Theorie oder die Geschlechtersoziologie kaum ernsthaft mit der Familie. ${ }^{20}$

Im Schnittfeld von Geschlechter- und Familientheorie stand vor Jahren die Thematisierung patriarchaler Strukturen. Heute behandelt kaum jemand in der Familiensoziologie diese Frage, im Unterschied zur Geschlechtersoziologie (Walby 1997; Connell 1987) oder Körpersoziologie (Turner 1984). Für die Familienforschung scheint die Frage entschieden, ob es noch patriarchale Strukturen gibt: Sie wird gar nicht mehr gestellt. Man könnte „patriarchale Strukturen“ als Konzept begreifen, mit dem „Ungleichheiten“ sowohl in der Familie als auch im Beruf erklärt werden könnten. Statt dessen herrscht weitgehend Konsens, dass in der Familie Gleichheit und Partnerschaftlichkeit durchgesetzt sei. ${ }^{21}$ Allerdings fällt es dann schwer, die weiterhin asymmetrische Aufgabenverteilung in Privathaushalten zu erklären. Kenntnisse der subtilen Mechanismen, die dazu führen, dass zum Beispiel der Anteil von Professorinnen oder von Unternehmensleiterinnen nach wie vor so gering ist, könnten aber durchaus auch theoretisch nutzbar gemacht werden für das Verständnis der scheinbar so schwer erklärbaren Tatsache, dass in den meisten Paarbeziehungen/Familien die Männer die bessere Berufsposition einnehmen. Häufig wird hier, in Ermangelung einer soziologisch adäquaten Theorie, auf die ökonomische Ressourcentheorie zurückgegriffen, der zufolge es gewissermaßen eine rationale Entscheidung innerhalb der Familie ist, wenn die Frau auf die Ausübung ihrer beruflichen Tätigkeit verzichtet bzw. diese reduziert, da dadurch für die Familie insgesamt mehr zu gewinnen ist als wenn die Frau auf ihren Ansprüchen beharren würde. Neben einfachen ad-hoc-Erklärungen (,die Männer wollen ihre Privilegien nicht abgeben“) kommen zunehmend wieder biologistische Erklärungen zum Zuge. Mit einem alten Patriarchats- oder Machtbegriff kommt man heute natürlich nicht mehr weit. Nur ist es theoretisch genauso unbefriedigend, mehr oder weniger selbstverständlich von Gleichheit und Partnerschaftlichkeit auszugehen - ohne die empirisch deutlichen Asymmetrien noch theoretisch fassen zu können.

20 Vgl. etwa Heintz (2001). Dieser Sammelband zur Geschlechtersoziologie mit etwa 20 Beiträgen hat seinen Schwerpunkt auf dem Zusammenhang von Geschlecht und sozialer Ungleichheit in der öffentlichen Sphäre (Bildung, Beruf, Politik), Familie und Paarbeziehungen werden nur am Rand thematisiert.

21 Symptomatisch dafür ist vielleicht, dass der Buchtitel „Vom Patriarchat zur Partnerschaft“ (Mitterauer/Sieder 1984) als zentrale Formel für die Modernisierung der Familie gilt. 


\subsection{Politik, Geschichte, Kultur}

Drei weitere Felder seien noch kurz gestreift: Familienpolitik, historische Familienforschung und Kultur. Zum ersten Bereich gibt es zahlreiche Arbeiten, gerade in jüngerer Zeit. Zum zweiten scheint die Forschung ein wenig erlahmt. Beim dritten besteht weiterhin Forschungsbedarf.

Familienpolitik. Im Zuge der demographischen Krise und der Überforderung des Sozialstaates gewann Familienpolitik - allerdings in enger Verknüpfung mit Sozialpolitik (Rentenversicherung, Gesundheitssystem), Steuerpolitik (Steuern für Kinderlose?) und Frauenförderung wieder stärker an Bedeutung. ${ }^{22}$ Für die Familientheorie ist dies ein wichtiges Feld, gerade auch, weil die Beziehungen von Familie und Staat seit jeher spannungsvoll sind. Es geht immer um das Verhältnis von privater Freiheit und öffentlicher Fürsorge. Deshalb sind normative Implikationen von Theorie hier noch weniger vermeidbar als sonst. ${ }^{23}$ Familienpolitik steht heute im Spannungsfeld zwischen Bevölkerungspolitik (Fertilitätsförderung), Gleichstellungspolitik (Frauenförderung) und Familienförderung (Förderung der Lebensform Familie).

Historische Familienforschung. Die historische Familienforschung war für die Theoriebildung wichtig, weil sie dem Begriff der modernen Familie historische Plastizität geben konnte, weil sie bestimmte Mythen korrigieren konnte: beispielsweise den Mythos Großfamilie (Mitterauer/Sieder 1984); oder weil sie falsche Auffassungen über radikale historische Brüche korrigieren half. Inzwischen sind auch in diesem Bereich die Verbindungen zwischen den Forschungsfeldern lockerer geworden (Schmidt 2002: 345) - obwohl gerade im Zuge der Individualisierungsdebatte immer wieder deutlich wurde, dass Veränderungstendenzen leicht fehlinterpretiert werden können, wenn sie nicht im längerfristigen historischen Vergleich gesehen werden.

Während die Entstehungsgeschichte der modernen Familie in den Grundzügen geklärt ist, bewegt sich die Diskussion über einen möglichen Übergang zur postmodernen Familie immer noch auf dünnem Eis. In der deutschen Familienforschung wird der Begriff im Grunde nicht ernst genommen. ${ }^{24}$ Zwar hatte Edward Shorter schon in den 1970er Jahren seine historischen Analysen zur Geburt der modernen Familie mit einem Kapitel über die ,postmoderne Familie“ abgeschlossen; und in Deutschland hat ein umfangreicher Sammelband diese Diagnose aufgegriffen und diskutiert (Lüscher/Schultheis/Wehrspaun 1988). Doch seither ist an dieser Diskussionsfront hierzulande wenig passiert, im Unterschied zu den USA. In der Sache gibt es allerdings deutliche Überschneidungen zur Individualisierungsthese.

22 Zum Beispiel Leisering (1992), Kaufmann (2003).

23 In anderen Ländern hat die politische Seite oft stärker Berücksichtigung in der Familiensoziologie gefunden. Vgl. etwa Singly (1994), wo die Entwicklung der letzten hundert Jahre in Frankreich auf zwei miteinander verknüpfte Tendenzen zugespitzt wird: zum einen Privatisierung und Autonomisierung (Familie zunehmend als Schutzraum persönlicher Individualität), zum anderen wachsende Intervention des Staates.

24 Es gibt aber auch kaum fundierte Diskussionen darüber, ob alternative Angebote der Zeitdiagnostiker - Spätmoderne, high modernity, Zweite Moderne, reflexive Moderne - der Charakterisierung der gegenwärtigen und zukünftigen Familie eher angemessen wären. 
Mit der Unklarheit über einen möglichen Übergang von der modernen zur postmodernen Familie hängt auch eine Unsicherheit bezüglich der Frage zusammen, in welcher Weise das „bürgerliche“ Familienmodell noch Geltung hat. Natürlich sind Tendenzen, die eine deutliche Veränderung gegenüber der klassischen bürgerlichen Familie des 19. Jahrhunderts (Rosenbaum) und der ersten Hälfte des 20. Jahrhunderts ausmachen, nicht zu übersehen, etwa im Bereich der Geschlechtsrollen, der Erziehungspraktiken oder der Veränderungen durch Technisierung und Medialisierung. Umgekehrt kann man aber Tendenzen der Verstärkung „,bürgerlicher“ Züge konstatieren. So ist zum Beispiel die in der Literatur überall als Kennzeichen der modernen bürgerlichen Familie genannte Intimisierung und Emotionalisierung der familialen Beziehungen eher noch gesteigert worden; ebenso die Autonomisierung der Familienmitglieder, wie sie sich u.a. bei den Wohnverhältnissen bemerkbar macht. Mit dem Bürgertum beginnt die Individualisierung der Wohnräume, ein Prozess, der bis heute anhält. Schließlich ist auch die romantische Liebe weiterhin unangefochten gültig als wichtigste Legitimationsbasis für Paarbildung und Familienbildung. Und vielleicht haben die Tendenzen zur Selbstverwirklichung, die man in der Tradition der Bildung des bürgerlichen Subjekts verorten kann, die bürgerliche Familie - anders als Pessimisten wie Lasch, Bellah oder Sennett befürchteten - sogar eher erneuert als zum Verschwinden gebracht.

Kultur. In einem gewissen Kontrast zum vielfach konstatierten cultural turn hat sich die familientheoretische Forschung von der neueren Kultursoziologie eher wenig beeinflussen lassen - und die ältere Kultursoziologie, wie sie von Parsons entwickelt wurde, gilt, wie gesagt, als nicht mehr anschlussfähig. Eine kultursoziologische Praxistheorie im Anschluss u.a. an Goffman und Bourdieu (vgl. Reckwitz 2000) könnte für die Familienforschung fruchtbar gemacht werden, wenn man anerkennt, dass die Familie nicht bloß ein Beziehungsnetz von Akteuren oder gar eine Veranstaltung zur Durchsetzung rationaler Entscheidungen ist, sondern ein soziales Feld, in dem Ritualen und Emotionen, körperlichen Praktiken und einverleibten Routinen besondere Bedeutung zukommt. ${ }^{25}$ Ein anderer Aspekt von Kultur ist mit dem Diskursbegriff im Anschluss an Foucault erfasst, der in der qualitativen Sozialforschung neuerdings Beachtung findet. Mit Familiendiskursen oder Familienrhetorik hat sich in Deutschland von allem die Arbeitsgruppe um Kurt Lüscher befaßt, wenn auch mit etwas anderer Ausrichtung (Lüscher 1995).

Das Stichwort Kultur des Individualismus führt noch einmal zurück zur Individualisierungsdebatte. Obwohl diese Debatte einen großen Teil der familiensoziologischen Literatur der letzten Dekaden bestimmt hat, gibt es vergleichsweise wenige systematische Theorie-Arbeiten zum Begriff des Individualismus in seinem Verhältnis zur Familie. „Individualisierung“ wurde meist verstanden als Problem für die Familie: Der wachsende Individualismus führe dazu, dass Individuen allein wohnen, sich nicht mehr binden, sich schneller wieder trennen, sich stärker auf ihre individuelle Erwerbsarbeit konzentrieren als auf die Familie. In diesem Sinn wurde die strukturelle Seite der Freisetzung relativ ausführlich analysiert, vor allem als Krisenindika-

25 Die Arbeiten von Jean-Claude Kaufmann $(1994,1999)$ können als Beispiele für in diesem Sinn theoriegeleitete Studien gelten. Vgl. dazu auch unsere milieuvergleichende Studie (Koppetsch/Burkart 1999). 
tor für die Familie. In der Argumentation der Theorie funktionaler Differenzierung gibt es dagegen eine eher positive Interpretation von Individualisierung. Man geht hier davon aus, dass die moderne Gesellschaft Individuen braucht, die sich auch als solche verstehen, d.h. eine reflexive Identität entwickeln (Schimank 2002). Und vielleicht kommt der Familie ja zunehmend die Funktion zu, Personen zu Individuen zu machen und die Selbstthematisierung zu fördern (Burkart 2004).

\section{Zur Binnenstruktur der Familie}

Theoretische Überlegungen zur Binnenstruktur der Familie befassen sich mit der inneren Ausdifferenzierung des Familiensystems, mit dem Verhältnis Ehe, Familie, Haushalt und Verwandtschaft, mit dem Geschlechter- und Generationsverhältnis innerhalb der Familie, mit Kommunikationsformen und Interaktionsregeln.

\subsection{Familie, Haushalt, Verwandtschaft}

Die weitgehende Gleichsetzung von Haushalt und Familie in der amtlichen Statistik hat $\mathrm{zu}$ vielen Missverständnissen in der öffentlichen Wahrnehmung der Prävalenz von Normalfamilien geführt - und zu Fehldarstellungen, die besonders im Wissenschaftsjournalismus verbreitet sind. So findet sich zum Beispiel immer wieder einmal die Aussage, in deutschen Großstädten gäbe es schon mehr als 50 Prozent Singles hier liegt offenkundig eine Verwechslung von Haushalten und Personen zugrunde, denn das relevante Bezugsdatum sind 50 Prozent Einpersonenhaushalte. Zumindest außerhalb der Familienforschung werden solche Aussagen auch von manchen Wissenschaftlern übernommen. Selbst in der Familiensoziologie kann man gelegentlich lesen, die klassische Normalfamilie sei bloß noch eine Minderheit. Man bezieht sich dabei auf die Haushaltsstatistik und zieht daraus den falschen Schluss, dass „Ehepaare ohne Kinder im Haushalt“ nicht zu den Familien zählen. Richtig ist allerdings, dass Familienhaushalte (d.h. Haushalte, in denen zwei Generationen zusammenleben) nicht mehr die größte Gruppe unter den Haushalten sind; ein Umstand, der auf das neolokale Prinzip der Familiengründung, die gestiegene Lebenserwartung und den hohen Anteil von Einpersonenhaushalten Älterer zurückzuführen ist. Musste wirklich erst die neuere Generationenforschung zeigen, dass Familien auch dann noch Familien sind, wenn die Generationen nicht alle unter einem Dach wohnen? (,multilokale Mehrgenerationenfamilie“; Bien/Marbach 1991; Bertram 2000) ${ }^{26}$

26 Anders wiederum ist die Lage, wenn man sich bei der Aussage, die „klassische Familie“ sei nur noch eine Minderheit, auf das Kriterium der traditionellen Rollentrennung bezieht. Dann wäre die „klassische Normalfamilie“ die Alleinverdiener-Familie (breadwinner) caretaker, die Ehefrau als Hausfrau und Mutter), und diese ist in dieser strikten Form in der Tat nur noch eine Minderheit. 
Es herrscht eine gewisse Unsicherheit über die erweiterte Kernfamilie. Die Regeln, wer dazu gehört, scheinen in der Tat stark aufgeweicht. Vielleicht sollte man in der Familienforschung wieder stärker auf kulturelle Aspekte (Rituale, symbolische Praktiken) achten, zum Beispiel die Bedeutung von Familienfesten, bei denen Verwandte zusammenkommen, die in alle Winde zerstreut sind, aber sich offenbar doch der einen „Familie“ oder „Sippe“ (Verwandtschaftssystem) zugehörig fühlen. Man könnte dann Kriterien finden, die nicht mehr auf formale Verwandtschaftsregeln beschränkt wären, ohne deswegen gleich alle familialen Beziehungen der individualisierten Beliebigkeit anheim zu stellen. Die Grenzen, wer noch dazu gehört und wer nicht (und vor allem: mit wem man etwas zu tun haben will oder nicht), waren auch in der Vergangenheit nicht so starr, wie die beliebte Rede von der ,Wahlverwandtschaft“ heute suggeriert. Die zahlreichen Stellungnahmen zur „Pluralisierung von Lebensformen“, „Auflösung des Familienverbands“ helfen hier jedenfalls nicht weiter. Dagegen könnten die aus der Ethnologie und der kulturvergleichenden Familienforschung bekannten Begriffe als Beschreibungsvokabular wieder stärker genutzt werden.

Die in weiten Teilen Mitteleuropas und der westlichen Welt vorherrschende Familienform, so lässt sich der Stand der Theorie vielleicht zusammenfassen, ist die Mehrgenerationen-Familie in Form des Generationsverbundes (Nave-Herz 2004: 32). ${ }^{27}$ Die einzelnen Generationen bilden relativ autonome Einheiten (Neo- bzw. Multilokalität, d.h. die Kinder-Generation lebt nur bis zur Gründung eines eigenen Haushalts bei den Eltern), die Ehe/Paarbeziehung hat Priorität gegenüber der Herkunfts- oder Abstammungsfamilie. Weitere Merkmale sind: Egalität beider Herkunftsfamilien, keine oder nur schwache Patrilinearität, neuerdings sogar Tendenzen der Matrilinearität (aufgrund der Zunahme von alleinerziehenden Müttern und multiplen Vaterschaften).

Bis in die 1970er Jahre wurde die von Parsons stammende Isolationsthese diskutiert (,strukturelle Isolation der Kernfamilie“). Es ging dabei um die relative Autonomie der Familie, um den Grad der Autonomie der Kleinfamilie im Verwandtschaftssystem. Sie wurde oft missverstanden und als widerlegt angesehen, nachdem gezeigt worden war, dass die Kontakte zwischen den Generationen und innerhalb der Verwandtschaft durchaus noch hoch waren. Schon in den 1950er und 1960er Jahren hatte es Studien gegeben, die zeigten, dass „Isolation“ nicht notwendigerweise weniger Kontakte zwischen den Generationen bedeuten muss, aber eine Lockerung von Verwandtschaftsbindungen nach sich zieht. Die theoretischen Anstrengungen zum Verhältnis von Familie und Verwandtschaft und zur Struktur der Verwandtschaft haben merklich nachgelassen. Spätestens seit Frank Furstenbergs Diagnose der „,conjugal succession“ (1987) - bei jeder neuen Ehe wechselt man einen Teil der Verwandtschaft aus - hält man in der von der Individualisierungsthese beherrschten Familiendiskussion Beziehungen zu Verwandten für eine Sache der freien Entscheidung, Wahl-Verwandtschaft eben. ${ }^{28}$ Zum Teil ist in der Theorie an die Stelle von Ver-

27 Generationsverbund in Abgrenzung zur Abstammungsfamilie, wo Patrilinearität und Priorität der Herkunftsfamilie gelten.

28 Eine der wenigen ausführlichen Publikationen zur Verwandtschaftsthematik: Schütze/Wagner 1998 . 
wandtschaft das Netzwerk getreten. In den 1980er und 1990er Jahren wurden zunehmend ,informelle“ Netzwerke untersucht, nicht nur im Zusammenhang mit dem Rückgang innerfamilialer Bindungen, sondern auch dem vermeintlichen Rückzug des Staates aus der Familienversorgung. In der Familienforschung wurden Netzwerke vor allem als Unterstützungssysteme betrachtet, als positiv funktionierendes Netz von Sozialkontakten. Seit Mitte der 1990er Jahre kam eine stärker problemorientierte Sichtweise hinzu, in der zum Beispiel Ambivalenzen (Lüscher/Pillemer 1998) und Konflikte herausgearbeitet wurden (Schmidt 2002: 69 ff.).

\subsection{Paarsystem, familiale Dynamik und Lebenszeit}

Zunächst ist theoretisch keineswegs trivial, dass die Paarbildung ebenso wie die Familiengründung eine neue Realität sui generis schafft, eine neue soziale Entität (Berger/Kellner 1965; Alberoni 1984). Ehe und Familie sind deshalb mehr als eine geregelte Kooperation oder eine einfache Beziehung zweier Individuen. Das gilt auch, wenn bestimmte institutionelle Rahmenbedingungen geschwächt sind, wie bei nichtehelichen Lebensgemeinschaften. Mit Paarbildung und Familiengründung entsteht ein neues Gebilde mit Systemcharakter - auch im Sinne einer Kultur (,Semantik“ in Luhmanns Terminologie): Die Gemeinsamkeit des Paares und der Familienmitglieder schafft eine neue Sinnebene. ${ }^{29}$ Unterschätzt wird in manchen Debatten der Charakter der Ehe als einer immer noch starken Institution im umfassenden Sinn (rechtlich, sozial, kulturell). Wer heiratet, erzeugt nach innen und nach außen eine höhere Verbindlichkeit und Verpflichtung, als wer nur „so“ zusammenlebt oder getrennt wohnt. Die neu entdeckte Bedeutung von Ritualen und Familienfesten ist ein Indikator dafür (Nave-Herz 2004: 138 ff.).

Parsons hat bereits früh die Familie als System mit einer Binnendifferenzierung analysiert (Parsons/Bales 1955). Einige Autoren aus dem Lager der Theorie der funktionalen Differenzierung sehen heute zunehmende Probleme der Entkopplung der Subsysteme, der mangelnden Integration, vor allem zwischen dem Eltern-KindSystem und dem Elternpaar-System (Filiation und Konjugalität; Herlth/Tyrell 1994). Meyer (1992) macht drei Teilsysteme bzw. „Privatheitstypen“ aus: partnerschaftsorientiert, kindheitsorientiert und individualistisch. Dahinter steckt auch die Auffassung, dass Ehe und Familie nicht mehr selbstverständlich zusammengehören; mehr noch: dass Liebe, Paarbeziehung, Ehe und Familie nicht mehr eine Einheit bilden. Daher die Auffassung in Teilen der Familiensoziologie, dass diese Bereiche auch eigenständig beforscht werden müssten.

Luhmann hat den temporalen Charakter von Systemen betont. Auch Familie ist ein System in der Zeit, ein dynamisches System. Während die Berücksichtigung der Lebenszeitperspektive von Individuen aufgrund der Erfolgsgeschichte von Lebenslauf- und Biographieforschung (Kohli 1985) längst selbstverständlich ist, gilt dies für

29 Eine neue Realitätsebene konstatiert auch Huinink (1995) mit der dialogischen Beziehung des Paares (vgl. dazu auch Nave-Herz 2004: 151). Im Methodologischen Individualismus wird dagegen tendenziell so getan, als handle es sich bei einem Paar um zwei unabhängige Individuen, die miteinander kooperieren oder sich austauschen. 
das Familiensystem noch nicht in gleichem Maße. Doch das theoretische Interesse ist auch in der Familienforschung in verschiedener Hinsicht zunehmend auf die Kategorie der Zeit gelenkt worden. Neben der historischen Familienforschung und der lebenszeitlichen Perspektive finden etwa die Problematik der Zeitstruktur und Zeitorganisation in Familienhaushalten Beachtung (Daly 1996; Hochschild 1997). Die temporale Dimension wurde insbesondere für einzelne Phasen des „Familienzyklus“ analysiert: von der Ablösung der Individuen von der Herkunftsfamilie über die Paarbildung und die Gründung einer eigenen Familie bis zur ihrer möglichen Auflösung.

\subsection{Paarbildung und Familiengründung}

Paarbildung. In der psychologischen Literatur, im Rahmen des Methodologischen Individualismus, aber auch allgemein in der Familienforschung ist meist von „Partnerwahl“" die Rede, ein Begriff, der struktursoziologisch irreführend ist, suggeriert er doch die merkwürdige Vorstellung, als wählte jedes Individuum aus einem mehr oder weniger großen Angebot einen Partner aus - und nur, wenn der andere zufällig den selben Partner aussucht, kommt es zur Paarbildung. Natürlich ist es richtig (und dies ist mit Partnerwahl oft auch gemeint), dass die Individuen selbst - und nicht wie früher deren Eltern - auf Partnersuche gehen oder mit der Bereitschaft in die Öffentlichkeit gehen, sich auswählen zu lassen. ${ }^{30}$ Aber insgesamt wäre hier doch eine strukturtheoretische oder interaktionistische Perspektive soziologisch treffender, deshalb spreche ich lieber von Paarbildung, verstanden als Ergebnis eines komplexen Prozesses der Interdependenzen von Wunschvorstellungen über ideale Partner, intentionalen Handlungen, zufälligen Begegnungen und einer Reihe von sozialen Strukturbedingungen. Die sozialen Regeln der Emergenz dieses Prozesses wären zu untersuchen, denn nicht durch zwei individuelle Wahl-Handlungen entsteht ein Paar, sondern durch eine Reihe von emotional und sexuell aufgeladenen Interaktionen in bestimmten sozialen und kulturellen Kontexten. ${ }^{31}$ Selbstverständlich gehören zu diesem Prozess auch individuelle Entscheidungen, etwa darüber, ob an kritischen Punkten der Weg zum Paar fortgesetzt werden soll oder nicht.

Ein Großteil der diesbezüglichen Forschungen kommt aus der Psychologie. Soziologische Analysen der Paarbildung konzentrieren sich gewöhnlich auf strukturelle Rahmenbedingungen und normative Regulierungen der auf Paarbildung bezogenen Interaktionsprozesse: Struktur von Heiratsmärkten, Gelegenheitsstrukturen, Homogamie- und Exogamie-Regeln. Aus der empirischen Forschung, die zeigt, dass es weiterhin soziale Regeln der Paarbildung gibt, seien drei markante Ergebnisse hervorgehoben, die auch theoretisch relevant sind: Erstens ist soziale Herkunft immer noch wichtig. Das verweist auf die Bedeutung des Habitus bei der Paarbildung

30 Wahrscheinlich wird man sogar sagen können, dass der Begriff „Partnerwahl“ früher bzw. in Kontexten von durch die Eltern arrangierten Ehen angemessener war als heute, weil die Wahl eines Ehepartners durch die Eltern eher eine Angelegenheit rationaler Wahl war als unter dem Zeichen von sexueller Attraktion und Liebe.

31 Hier sei auf die Studie über den Konsum der Romantik von Eva Illouz (2003) hingewiesen, die sich mit der Komplexität der Paarbildung im Kontext der Kulturindustrie befasst. 
(Bourdieu 1982: 373 ff.). Zweitens hat sich der Bildungsgrad als wichtiger Homogamie-Faktor in den Vordergrund geschoben. Und im Zusammenhang mit den Umwälzungen im Geschlechterverhältnis hat sich damit auch die Homogamie zwischen Partnern vergrößert. Diese Konstellation hat einen neuen Paartypus hervorgebracht: das egalitäre, partnerschaftlich orientierte, individualisierte Paar. Zwei seiner Ausprägungen sind für die Familientheorie besonders interessant: Das kinderlose Doppel-Karriere-Paar und - in noch stärkerer Abkehr vom Komplementaritätsmodell (das meist mit Hypergamie verbunden war) - die Paarbeziehung ohne gemeinsamen Haushalt (living apart together). ${ }^{32}$

Übergang in die Elternschaft, Familiengründung. Wann führt die Paarbeziehung in die Ehe, wann führt die Ehe in die Elternschaft? Wenn auch mit abnehmender Tendenz, so besteht in Deutschland immer noch eine starke Verbindung zwischen Eheschließung und Familiengründung. Die Frage der Entscheidung zur Elternschaft wird weiterhin diskutiert (Schneider/Matthias-Bleck 2002). Doch ist in den letzten Jahren zunehmend bemerkt worden, dass es für einen wachsenden Anteil der Bevölkerung nicht zur Familiengründung kommt: Die Zahl von Singles und Kinderlosen steigt. Erklärungsversuche für Kinderlosigkeit setzen im Allgemeinen am Vereinbarkeitsproblem, der Bildungsexpansion und den Umwälzungen im Geschlechterverhältnis an (Berger/Kahlert 2006; Kreyenfeld/Konietzka 2006). ${ }^{33}$

Paarbildung und Familiengründung sind Elemente des Zusammenhangs von Lebenslauf und Familiendynamik. Die Forschung hat sich inzwischen weiter ausdifferenziert, Kindheit, Jugend und Alter sind ebenso eigene Forschungsfelder geworden wie Lebenslauf- und Biographieforschung. In diesem Zusammenhang stellt sich die Frage, ob Ehe und Familie aus der Sicht der Lebenszeit des Einzelnen noch den Charakter einer dauerhaften, lebenslangen Lebensform haben oder ob sie nur noch Lebensphasen sind. Dafür spricht manches (Burkart 1997). Doch nach wie vor gilt die Unkündbarkeit der Elternschaft, und diese gilt lebenslang, d.h. auch für die alt gewordenen Eltern - und deshalb sind auch für die erwachsenen Kinder die Beziehungen zur Herkunftsfamilie im kulturellen Normalfall stabil.

\subsection{Sozialisation und Generationsbeziehungen}

Sozialisation. Das Generationsverhältnis in der Familie konzentriert sich zunächst, in der neolokalen Kernfamilie, auf das Verhältnis der jungen Eltern zu ihren Kindern. Dies ist das Feld für die Sozialisationsforschung, die in den 1970er Jahren eine Blütezeit hatte, danach aber allmählich aus dem Bereich der Familiensoziologie verschwand (Schmidt 2002: 204 ff.). Das ist um so erstaunlicher, als von den wenigen Funktionen, die der Familie noch geblieben sind, die Sozialisation der Kinder die wohl wichtigste ist. Die Soziologie hat sich aus diesem Forschungsfeld weitgehend

32 Abgesehen von dieser Form des freiwillig Getrenntlebens haben auch andere mobile Lebensformen (etwa durch Berufspendeln) an Bedeutung gewonnen (Schneider/Limmer/ Ruckdeschel 2002).

33 Dabei richtet sich der Blick aber überwiegend auf die Frauen. Tölke/Hank (2005) sprechen deshalb von den Männern als „vernachlässigtes Geschlecht““. 
zurückgezogen und es der Entwicklungspsychologie, der Sozialpsychologie und der pädagogisch orientierten Sozialisationsforschung überlassen. ${ }^{34}$ Und die soziologische Kindheitsforschung befasst sich weniger mit familialer Sozialisation als mit der Lebenswelt von Kindern. Für die Soziologie wäre aber wichtig zu wissen, welchen Beitrag die Familie noch zur Persönlichkeitsentwicklung leistet, zur Entwicklung des moralischen Bewusstseins, zur Identitätsbildung, zur Subjektivierung. Schließlich verweisen die wachsenden Probleme, die sich an den Schulen zeigen, immer noch stark auf den familialen Hintergrund.

Auszug aus dem Elternhaus. Neben der Kindheits- hat sich auch die Jugendforschung gegenüber der Familienforschung verselbständigt. Sie ist jedoch gerade für die Auflösung des Familienhaushalts wichtig: Wann und unter welchen Umständen verlassen die Kinder das Elternhaus? Als Gegentrend zur Auflösung von Familien und zur Individualisierung wird seit einiger Zeit das Phänomen registriert, dass Töchter und vor allem Söhne bis weit ins Erwachsenenalter zuhause wohnen bleiben („Hotel Mama“). Und dies bei weiterhin vorgerücktem Pubertätsalter, so dass die Lebenszeit, die man als Jugendlicher im Elternhaushalt verbringt, weiter steigt.

Altern und Generationsbeziehungen. Auch die Alternsforschung hat sich institutionell ausdifferenziert. Gleichwohl gibt es einen Bereich, wo Alterns- und Familienforschung noch eng zusammenarbeiten: Die familiale Generationsforschung hat sich in den letzten Jahren zu einem neuen Schwerpunkt entwickelt (Lüscher/Schultheis 1993; Kohli/Szydlik 2000). Sie hat den Blick für die Tatsache geschärft, dass Familie nicht umstandslos mit Haushalt gleichgesetzt werden kann; sie hat deutlich gemacht, dass das Verhältnis der Generationen nicht so schlecht ist, wie man es aufgrund mancher Argumente aus dem Kontext der Individualisierungsdebatte erwarten könnte. Die Austauschprozesse, Kontakte und Transfers (Geld und Dienstleistungen) sind vielfältig und reichhaltig. Aber es wurde auch deutlich, dass unter den veränderten demographischen und politischen Bedingungen die mittlere Generation von zwei Seiten unter Druck gerät, weshalb auch von einer ,Sandwich"-Generation die Rede ist: Finanzierung und Erziehung der eigenen Kinder auf der einen Seite, Pflege der alten Eltern auf der anderen Seite.

\subsection{Geschlechterverhältnis innerhalb der Familie}

Das Geschlechterverhältnis innerhalb der Familie weist einige Besonderheiten auf und kann deshalb nicht analog zum Geschlechterverhältnis auf der Makro-Ebene untersucht werden. Es geht hier ja nicht um das Verhältnis von Männern und Frauen, sondern um das Paar als einer besonderen Beziehung zwischen zwei Personen, um Liebe, emotionale Unterstützung und Partnerschaft. Das hat Auswirkungen auf die Arbeitsteilung und Kooperation zwischen Mann und Frau in Ehe und Familie, hinsichtlich der Hausarbeit und der Kindererziehung, aber auch bezüglich der Frage, wo die Familie ihren Standort hat, wenn Mann und Frau nicht in der selben Stadt arbei-

$34 \mathrm{Zu}$ den wenigen Ausnahmen gehören Leu/Krappmann (1999), Grundmann (1999) oder Hopf (2005). 
ten. Zur Arbeitsteilung im Haushalt gibt es zahlreiche empirische Studien. Abgesehen davon, dass sie ein überraschendes Ausmaß weiterhin bestehender Geschlechtsunterschiede dokumentieren, wurde auch deutlich, dass keineswegs in allen Milieus das Ideal der egalitären Ehe vorherrscht. ${ }^{35}$ Aber das Begriffsinstrumentarium zur Typologisierung von Geschlechterverhältnissen beschränkt sich häufig auf einfache Dichotomien wie traditional vs. egalitär - und mehr oder weniger diffuse Zwischenkategorien.

Die Frage, wie die Arbeitsteilung organisiert ist, hängt vom Charakter der Paarbeziehung und ihren Regulativen (Regeln, Normen, Beziehungsidealen) ab. Drei Grundtypen lassen sich unterscheiden: Arbeitsteilung aufgrund von unterschiedlichen Ressourcen oder Machtverhältnissen; partnerschaftliche Aushandlung der Arbeitsteilung; und Arbeitsteilung aufgrund traditioneller Geschlechtsrollen in Verbindung mit Liebe oder aufgrund von Habitualisierungen und Routinen. Was den ersten Punkt betrifft, hat es seit der Diskussion um die Ressourcen-Theorie (Held 1978) wenig Neues gegeben. Seither stehen eher Studien zur partnerschaftlichen Aushandlung im Vordergrund.

Mann und Frau in der Ehe unterscheiden sich natürlich nicht nur in Bezug auf Tätigkeiten (Arbeitsteilung, Alltagsorganisation), sondern auch in Bezug auf ihre wichtigsten Rollen innerhalb der Familie, die Elternrollen. Auch hier wird ein grundsätzlicher Wandel diskutiert, das Auftauchen von „,neuen Vätern“. Es scheint festzustehen, dass Väter heute stärkere affektive Bindungen zu ihren Kindern haben. Aber hat sich deshalb die Vaterrolle grundlegend gewandelt? Fthenakis/Minsel (2001) etwa glauben, feststellen zu können, dass dies nicht der Fall ist. Zwar wurde immer wieder das Aufkommen eines neuen Vatertypus konstatiert, eines Außenseitertypus, mit dem man die Hoffnung verband, er würde im Zuge der allgemeinen Umwälzungen im Geschlechterverhältnis komplementär zur Veränderung der Frauen- und Mutterrolle auch die Familienstruktur auf eine neue Basis stellen. Diese Hoffnungen haben sich bis heute nicht erfüllt, zumindest nicht auf der Ebene der Praxis (zum Beispiel gemessen an der Zeit, die Väter und Müttern mit ihren Kindern verbringen). Statt dessen ist häufiger vom „Verschwinden des Vaters“ oder von „shrinking of fatherhood“ (Jensen 1999) die Rede, zumindest von einer Fragmentierung der Vaterschaft (Vaskovics 2002).

Liebe und Partnerschaft. Wenn sich die Geschlechtersoziologie dem Thema Liebe nur zögernd nähert, dann liegt das daran, dass in feministischer Sicht Liebe häufig zur Verschleierung männlicher Herrschaft diente. Die Familienforschung hat aus anderen Gründen das Thema Liebe vernachlässigt (Burkart 1998). Vielfach wurde konstatiert, dass sich das bürgerliche Modell der Liebesehe - verstanden als Junktim zwischen Liebe und Ehe: wenn man liebt, sollte man heiraten; wenn man heiratet, sollte man sich lieben - spätestens im 20. Jahrhundert in allen Schichten durchgesetzt hat. Genauso wurde aber auch deutlich, dass die Liebesehe aus sich heraus Probleme mit der Stabilität bekommt, weil die Liebe als Basis für Dauerhaftigkeit nicht taugt. Als eine mögliche Lösung dieses Problems wurde - schon im 19. Jahrhundert

35 Koppetsch/Burkart (1999), Hopf et al. (2001), Huinink/Röhler (2005). Vgl. auch die zahlreichen Hinweise auf einen ,,vollzogenen normativen Einstellungswandel“ bei immer noch starken faktischen Unterschieden (z.B. Nave-Herz 2004: 155). 
- das Prinzip der Partnerschaftlichkeit entwickelt (Leupold 1983). Inzwischen glauben viele Autoren, dass es die Liebe als Regulativ von Ehe und Paarbeziehung abgelöst habe (Giddens 1993). Allerdings gibt es auch Skepsis gegenüber diesem Prinzip, weil es zu rationalistisch sein könnte (Hochschild 2003; Burkart/Koppetsch 2004). ${ }^{36}$

Auflösung von Paarbeziehung und Familie. Die zeitliche Perspektive ist auch deshalb wichtiger geworden, weil die Familie nicht länger eine unauflösliche Einheit ist. Die Scheidungsforschung ist zu einem Schwerpunkt der Forschung geworden, an dem sich auch die Reichweite und Erklärungskraft der Theorie-Ansätze gut durchspielen ließe. Aus dem Umfeld der Mannheimer Scheidungsstudie (Esser 1999, 2001; Klein/Kopp 1999) und einem neuen Schwerpunktprogramm zur Beziehungsund Familienentwicklung (Feldhaus 2005) gibt es Ansätze für ein integriertes Modell der Stabilität bzw. Instabilität von Paarbeziehungen, bei dem neben der Handlungsebene und der Gelegenheitsstruktur auch Tendenzen wie Wertewandel, Individualisierung und gemeinsame Sinnwelt betont werden.

\subsection{Familie als Kommunikationssystem und Gefühlsgemeinschaft}

Häufig wird konstatiert, dass der spätmodernen Familie neben der Aufgabe, Kinder in die Welt zu setzen und deren Persönlichkeit in den Grundzügen zu formen (Reproduktion der Gattung und Sozialisation), noch die Funktion verblieben sei, den Kindern aber auch den Erwachsenen ein Milieu zur Verfügung zu stellen, in dem sie Geborgenheit, Wärme und emotionale Sicherheit erleben und erfahren können (soziale Reproduktion). Die Soziologie hat diese Aspekte oft nur in sehr allgemeiner Form thematisiert und die Details - etwa zu den Faktoren, wie in einer Familie ein emotionales Klima hergestellt wird - der Psychologie überlassen. Emotionen seien kein legitimer Gegenstand der Soziologie, so glauben immer noch viele. Aber ohne eine affektiv-rituelle Basis, das ist seit Tönnies und Durkheim bekannt, ist der Aufbau von Solidargemeinschaften kaum möglich (Huinink/Strohmeier/Wagner 2001).

Folgt man Luhmann, dann sind alle sozialen Systeme Kommunikationssysteme. Im Unterschied zu anderen zeichnet sich das spezielle soziale System Familie ihm zufolge durch eine besondere Form der „enthemmten Kommunikation“ (Luhmann 1990: 203 ff.) aus, bei der fast alles thematisierbar sei. Zweifellos ist das familiale Gespräch wichtig, wie auch Berger/Kellner (1965) in einem einflussreichen Aufsatz betonten, in dem sie vor allem die identitätsstabilisierende Funktion der Ehe hervorgehoben hatten. ${ }^{37}$ In diesem Zusammenhang sind auch die Untersuchungen zu Konsens-Fiktionen wichtig (Hahn 1983). Aber familiale Kommunikation ist nicht unbedingt auf Konsens angelegt, im Gegenteil: Folgt man Luhmann, ist die Kontinuität der Kommunikation auf das Nein-Sagen angewiesen; und in einer konflikttheoretischen Perspektive sind gerade Konflikte und Dissens wichtig für Gemeinschaftsbil-

36 Zur Verschränkung von Ökonomie und Emotionen (oder Geld und Liebe) siehe auch Wimbauer (2003) und Illouz (2003).

37 Untersuchungen wie jene von Angela Keppler (1994) über Tischgespräche sind selten geblieben. Sie wurde in der Familiensoziologie auch wenig rezipiert. 
dung. Deshalb könnte auch die Untersuchung von Dissensfiktionen fruchtbar sein (Hildenbrand 2006).

\section{Die Theorie-Zukunft der Familiensoziologie und die Zukunft der Familie}

Es mag sein, dass dieser Bericht Defizite und Desiderata der Familienforschung überbetont und damit die zweifellos vorhandenen Erfolge und Erträge vernachlässigt hat. Wissenschaftliche Berichterstattung gerät zunehmend in das Dilemma zwischen einer durch die neuen Evaluations- und ranking-Mechanismen geforderten Selbstanpreisung und der immer noch gültigen Maxime, dass wissenschaftlicher Fortschritt am besten durch Kritik und Selbstkritik zustande kommt. Die Leistungen auf vielen Teilgebieten der Familienforschung sind ja nicht zu übersehen. Aber wenn die hier vorgenommene Diagnose richtig ist, dann besteht eines der Kernprobleme der Forschung in der Ausdifferenzierung von Forschungsfeldern und damit der wachsenden Schwierigkeit, noch eine theoretische Integration zu erreichen. Darüber hinaus sehen sich viele Forschungsfelder mit neuen Herausforderungen konfrontiert.

In diesem Sinn sollen abschließend einige Aufgaben für die Zukunft knapp umrissen werden. Seriöse Zukunftsüberlegungen bewegen sich meist in den Bahnen der bisherigen Diskussionen, wie sich auch auf einer der Zukunft der Familie gewidmeten Jahrestagung der Sektion Familiensoziologie (2005 in Lüneburg) zeigte. Eine der dort meistdiskutierten Tendenzen war die Vermutung einer weiteren Fragmentierung der Elternschaft, insbesondere der Vaterschaft. Ein anderer Aspekt war die Technisierung des Haushalts, deren soziale Folgen aber noch unklar sind. In Bezug auf die Zukunft der Pflege machen sich Alternsforscher Sorgen. Aber in theoretischer Hinsicht ist die Zukunft schwer zu prognostizieren. Die postmoderne Familie bleibt eine vage Vorstellung, und auch die weitere Entwicklung der Individualisierung ist offen.

Auf eine wichtige Herausforderung nicht nur für die Familienforschung, sondern für die Soziologie insgesamt sei abschließend noch etwas ausführlicher hingewiesen. Gemeint ist das Rahmenthema des diesjährigen Soziologie-Kongresses, „Die Natur der Gesellschaft“, wo es um die Herausforderung der Sozial- und Kulturwissenschaften durch die „Lebenswissenschaften“ (Hirnforschung, Genetik, Molekularbiologie usw.) geht. Die Erfolge dieser Forschungsrichtungen betreffen auch das Thema Familie und private Lebensformen. Eine Re-Naturalisierung des privaten Lebens, eine Biologisierung von familialen und von Geschlechtsrollen, könnte die Debatte der nächsten Jahre und Jahrzehnte bestimmen. Schon heute kommt in der Öffentlichkeit zunehmend eher jenen Argumenten Überzeugungskraft zu, die sich statt auf Soziologie (Rollen, Normen, Strukturzwänge etc.) auf Biologie (Gene, Hormone, Gehirnregionen) beziehen. Es ist unübersehbar, dass in der Wissenschaftspublizistik, von der man annehmen darf, dass sie für das Bewusstsein der Zeitgenossen großen Einfluss hat, immer häufiger Ergebnisse und Thesen verbreitet werden, denen zufolge biologische Faktoren (Gene, Hormone) wichtiger sind als Erziehung, soziale Umwelt oder kulturelle Prägungen. Das gilt etwa für Themen wie Liebe, Sexualität und Partner- 
wahl, kindliche Entwicklung, Mutter-Kind-Beziehung, Geschlechtsunterschiede, körperlich-emotionale Eigenschaften, Altern, Körpergröße oder Lebenserwartung. Etwas überspitzt gesagt: Während wir uns zum Beispiel intern noch streiten, wie rational und wie individuell die Partnerwahl ist - ob sie eine Entscheidung ist, ob sie eine rationale Entscheidung ist - berichten die Zeitungen fast täglich von neuen Ergebnissen der Hirnforschung oder Genetik, mit denen auch die Paarbildung auf eine solide biologische Grundlage gestellt werden könne.

Für den Bereich der Familienforschung ergibt sich daraus in gewisser Weise die paradoxe Situation, dass wir es auf der einen Seite mit einer Stärkung biologistischer Interpretationen von Lebensformen und familialem Zusammenleben (Verhältnis der Geschlechter, Eltern-Kind-Verhältnis, Sexualität) zu tun haben, auf der anderen Seite aber Lebensweisen und die Formen familialen Zusammenlebens von der biologischen Basis immer mehr gelöst werden: Biologische und soziale Elternschaft (vor allem Vaterschaft) fallen immer häufiger auseinander, die Reproduktion wird weiter von der Natur gelöst (Reproduktionsmedizin, Gentechnologie), es gibt Tendenzen der Professionalisierung der Mutterschaft (Leihmütter, Tagesmütter); Blutsverwandtschaft wird relativ zu Wahlverwandtschaft unwichtiger, die starken intergenerationellen Veränderungen der Körpergröße, des Körpergewichts und der Lebenserwartung sind kaum allein biologisch zu erklären, ebensowenig wie die Unterschiede in der Präsentation der Geschlechter (doing gender), die markanter geworden sind.

Geschlecht, Körper, Sexualität, Liebe, Geburt, Gesundheit, Altern und Tod - dies sind zentrale Schnittstellen von Natur und Kultur, an denen heute die Definitionskämpfe zwischen Soziologie/Kulturwissenschaften und Biologie/Lebenswissenschaften ausgetragen werden. Diese Schnittstellen sind in vielfältiger Weise Gegenstand oder Ausgangspunkte familiensoziologischer Forschung, betreffen aber auch andere Forschungsfelder. Der Familiensoziologie könnte insofern in Zukunft wieder eine zentrale Rolle zukommen, wenn es ihr gelingt, diese zahlreichen Spezialgebiete der Forschung unter dem weiten Dach einer gewissermaßen multilokalen Familiensoziologie zusammenzuhalten und die theoretischen Stützpfeiler dieser offenen Architektur gelegentlich zu restaurieren.

\section{Literatur}

Alberoni, F. (1984). Movement and institution. New York: Columbia University Press.

Allert, T. (1998). Die Familie. Fallstudien zur Unverwüstlichkeit einer Lebensform. Berlin: de Gruyter.

Apple, R. D. (2006). Perfect motherhood. Science and childrearing in America. Fredericksburg: Rutgers University Press.

Baber, K. M./Allen K. R. (1992). Women and families. Feminist-reconstructions. New York: Guilford.

Beck, U./Beck-Gernsheim, E. (1993). Nicht Autonomie, sondern Bastelbiographie. Anmerkungen zur Individualisierungsdiskussion am Beispiel des Aufsatzes von Günter Burkart. Zeitschrift für Soziologie, 22, S. 178-187.

Beck, U./Beck-Gernsheim, E. (Hrsg.) (1994). Riskante Freiheiten. Frankfurt/M.: Suhrkamp.

Bellah, R.N. /Madsen, R./Sullivan, M. W./Swidler, A./Tipton, S. M. (1985). Habits of the heart. Individualism and commitment in American life. Berkeley: University of California Press. 
Benninghaus, C. (2005). Einleitung [zum Schwerpunktthema Kinderlosigkeit]. Feministische Studien, 23, S. 3-8.

Berger, P. A./Kahlert, H. (Hrsg.) (2006). Das „Problem“ des Demographischen Wandels und die Modernisierung der Geschlechterverhältnisse als Lösung? Frankfurt/M.: Campus.

Berger, P. L./Kellner, H. (1965). Die Ehe und die soziale Konstruktion der Wirklichkeit. Soziale Welt, 16, S. 220-235.

Bertram, H. (1995). Soziologische Familienrhetorik. In: Vaskovics, L. A. (Hrsg.) (1995). Familie - Soziologie familialer Lebenswelten. München: Oldenbourg (Soziologische Revue, Sonderband 3), S. 446-453.

Bertram, H. (2000). Die verborgenen familiären Beziehungen in Deutschland: Die multilokale Mehrgenerationenfamilie. In: Kohli, M./Szydlik, M. (Hrsg.). Generationen in Familie und Gesellschaft. Opladen: Leske + Budrich, S. 97-121.

Bertram, H. (2002). Intimität, Ehe, Familie und private Beziehungen. Soziale Welt, 53, S. 415-422.

Bien, W./Marbach, J. (1991). Haushalt - Verwandtschaft - Beziehungen. Familienleben als Netzwerk. In: Bertram, H. (Hrsg.). Die Familie in Westdeutschland. Stabilität und Wandel familialer Lebensformen. DJI: Familien-Survey 1. Opladen: Leske + Budrich, S. 3-44.

Blossfeld, H.-P./Müller, R. (1996). Sozialstrukturanalyse, Rational Choice Theorie und die Rolle der Zeit. Ein Versuch zur dynamischen Integration zweier Theorieperspektiven. Soziale Welt 47, S. 382-400.

Boltanski, L./Chiapello, E. (2003). Der neue Geist des Kapitalismus. Konstanz: Universitätsverlag (original: Le nouvel ésprit du capitalisme, Paris 1999).

Boss, P.G. et al. (Eds.) (1993). Sourcebook of family theories and methods. A contextual approach. New York: Plenum.

Bourdieu, P. (1982). Die feinen Unterschiede. Kritik der gesellschaftlichen Urteilskraft. Frankfurt/M.: Suhrkamp.

Bourdieu, P. (1998). La domination masculine. Paris: Seuil.

Bourdieu, P./Passeron, J.-C. (1971). Die Illusion der Chancengleichheit. Stuttgart: Klett.

Burkart, G. (1998). Auf dem Weg zu einer Soziologie der Liebe. In: Hahn, K./Burkart, G. (Hrsg.). Liebe am Ende des zwanzigsten Jahrhunderts. Opladen: Leske + Budrich, S. 1550.

Burkart, G. (2002). Stufen der Privatheit und die diskursive Ordnung der Familie. Soziale Welt, 53, S. 397-414.

Burkart, G. (2004). Selbstreflexion und Familienkommunikation. Die Kultur virtuoser Selbstthematisierung als Basis der Modernisierung von Familien. Familiendynamik, 29. Jg., Heft 3, S. 233-256.

Burkart, G. (2005). Die Familie in der Systemtheorie. In: Runkel, G./Burkart, G. (Hrsg.). Funktionssysteme der Gesellschaft. Beiträge zur Systemtheorie von Niklas Luhmann. Wiesbaden: VS Verlag für Sozialwissenschaften, S. 101-128.

Burkart, G./Koppetsch, C. (2001). Geschlecht und Liebe. Überlegungen zu einer Soziologie des Paares. In: Heintz, B. (Hrsg.). Geschlechtersoziologie. (Kölner Zeitschrift für Soziologie und Sozialpsychologie, Sonderband 41). Opladen: Westdeutscher Verlag, S. 431-453.

Burkart, G./Koppetsch, C. (2004). Die Ordnung des Paares und die Grenzen der Partnerschaft. Psychotherapie und Sozialwissenschaften - Zeitschrift für Qualitative Forschung, 6, S. 7388.

Busch, F. W./Nave-Herz, R. (Hrsg.) (2005). Familie und Gesellschaft. Beiträge zur Familienforschung. Oldenburg: BIS/Universität Oldenburg.

Cheal, D. (1999). The one and the many. Modernity and postmodernity. In: Allen, G. (Ed.). The sociology of the family. A reader. Oxford: Blackwell, p. 56-85 
Cherlin, A. J./Furstenberg, F. (1989). The changing European family: Lessons for the American reader. Journal of Family Issues, 9, p. 291-297.

Cohen, J. L./Arato, A. (1992). Civil society and political theory. Cambridge/Mass.: MIT Press.

Connell, R. W. (1987). Gender and power. Society, the person and sexual politics. Stanford: Stanford University Press.

Daly, K. J. (1996). Families and time: Keeping pace in a hurried culture. London: Sage.

De Singly, F. (1994). Die Familie der Moderne. Konstanz: UVK.

Doherty, W. J. (1999). Postmodernism and family theory. In: Sussmann, M. B./Steinmetz, S. K./Peterson, G. W. (Eds.) (1999). Handbook of marriage and the family. Second Edition. New York/London: Plenum, p. 205-217.

Esser, H. (1999). Heiratskohorten und die Instabilität von Ehen. In: Klein T./Kopp, J. (Hrsg.). Scheidungsursachen aus soziologischer Sicht. Würzburg: Ergon, S. 63-89.

Esser, H. (2001). Das „Framing“ der Ehe und das Risiko zur Scheidung. In: Huinink, J./ Strohmeier, K. P./Wagner, M. (Hrsg.). Solidarität in Partnerschaft und Familie. Zum Stand familiensoziologischer Theoriebildung. Würzburg: Ergon, S. 103-127.

Feldhaus, M. (2005). Beziehungs- und Familienentwicklungs-Panel. Erste Übersicht einer Gesamtkonzeption (DFG-Schwerpunktprogramm 1161). Manuskript.

Friedrichs, J. (Hrsg.) (1998). Die Individualisierungs-These. Opladen: Leske + Budrich.

Fthenakis, W. E./Minsel, B. (2001). Die Rolle des Vaters in der Familie. Stuttgart: Kohlhammer.

Furstenberg, F. F. (1987). Fortsetzungsehen. Ein neues Lebensmuster und seine Folgen. Soziale Welt, 38, S. 29-39.

Gelles, R. J. (1995). Contemporary families. A sociological view. Thousand Oaks: Sage.

Giddens, A. (1993). Wandel der Intimität. Sexualität, Liebe und Erotik in modernen Gesellschaften. Frankfurt/M.: Fischer.

Giddens, A. (1999). Runaway world. How globalization is reshaping our lives. London: Profile Books.

Goffman, E. (1994). Das Arrangement der Geschlechter. In: Goffman, E. Interaktion und Geschlecht. Frankfurt/M.: Campus, S. 105-158.

Grundmann, M. (1999). Konstruktivistische Sozialisationsforschung. Lebensweltliche Erfahrungskontexte, individuelle Handlungskompetenzen und die Konstruktion sozialer Strukturen. Frankfurt/M.: Suhrkamp.

Hahn, A. (1983). Konsensfiktionen in Kleingruppen. Dargestellt am Beispiel von jungen Ehen. In: Neidhardt, F. (Hrsg.) Gruppensoziologie. Perspektiven und Materialien. (Kölner Zeitschrift für Soziologie und Sozialpsychologie, Sonderband 25). Opladen: Westdeutscher Verlag, S. 210-232.

Heintz, B. (Hrsg.) (2001). Geschlechtersoziologie. (Kölner Zeitschrift für Soziologie und Sozialpsychologie, Sonderband 41). Opladen: Westdeutscher Verlag.

Held, T. (1978). Soziologie ehelicher Machtverhältnisse. Darmstadt und Neuwied: Luchterhand.

Herlth, A./Brunner, E. J./Tyrell, H./Kriz, J. (Hrsg.)( 1994). Abschied von der Normalfamilie? Partnerschaft kontra Elternschaft. Berlin: Springer.

Hildenbrand, B. (2006). Dissensfiktionen bei Paaren. In: Burkart, G. (Hrsg.). Die Ausweitung der Bekenntniskultur - neue Formen der Selbstthematisierung. Wiesbaden: VS-Verlag .

Hill, P. B./Kopp, J. (1995). Familiensoziologie. Grundlagen und theoretische Perspektiven. Stuttgart: Teubner.

Hochschild, A. R. (1989). The economy of gratitude. In: Franks, D. D:/McCarthy, E. D. (Eds.). The sociology of emotions. Original essays and research papers. Greenwich: JAI Press, p. 95-113.

Hochschild, A. R. (1997). The time bind. When home becomes work and work becomes home. New York: Holt. 
Hochschild, A. R. (2001). Globale Betreuungsketten und emotionaler Mehrwert. In: Hutton, W./ Giddens, A. (Hrsg.). Die Zukunft des globalen Kapitalismus. Frankfurt/M.: Campus, S. 157-176.

Hochschild, A. R. (2003). The commercialization of intimate life: Notes from home and work. Berkeley: University of California Press.

Hochschild, A. R. (2005). On the edge of the time bind. Time and market culture. Social Research, 72 , p. 339-355.

Hopf, C. (2005). Frühe Bindungen und Sozialisation. Eine Einführung. Weinheim/München: Juventa.

Hopf, C./Hartwig, M. (Hrsg.) (2001). Liebe und Abhängigkeit. Partnerschaftsbeziehungen junger Frauen. Weinheim: Juventa.

Höpflinger, F. (1997). Bevölkerungssoziologie. Eine Einführung in bevölkerungssoziologische Ansätze und demographische Prozesse. Weinheim: Juventa.

Huinink, J. (1995). Warum noch Familie? Zur Attraktivität von Partnerschaft und Elternschaft in unserer Gesellschaft. Frankfurt/M.: Campus.

Huinink, J. (2000). Bildung und Familienentwicklung im Lebensverlauf. Zeitschrift für Erziehungswissenschaft, 3, S. 209-227.

Huinink, J. (2002). Polarisierung der Familienentwicklung in europäischen Ländern im Vergleich. In: Schneider, N. F./Matthias-Bleck, H. (Hrsg.) (2002). Elternschaft heute. Gesellschaftliche Rahmenbedingungen und individuelle Gestaltungsaufgaben. (Zeitschrift für Familienforschung, Sonderheft 2). Opladen: Leske + Budrich, S. 49-74.

Huinink, J./Strohmeier, K. P./Wagner, M. (Hrsg.) (2001). Solidarität in Partnerschaft und Familie. Zum Stand familiensoziologischer Theoriebildung. Würzburg: Ergon.

Huinink, J./Röhler, K. A. (2005). Liebe und Arbeit in Paarbeziehungen. Zur Erklärung geschlechtstypischer Arbeitsteilung in nichtehelichen und ehelichen Lebensgemeinschaften. Würzburg: Ergon.

Illouz, E. (2003). Der Konsum der Romantik. Liebe und die kulturellen Widersprüche des Kapitalismus. Frankfurt/M.: Campus.

Jensen, A.-M. (1999). Partners and parents in Europe. A gender divide. In: Arnlaug L. (ed.). Family change. Practices, policies, and values (Comparative social research, Vol. 18). Stamford.: JAI, p. 1-29.

Katz, E. (1997). The intra-household economics of voice and exit. Feminist Economics, 3, p. $25-46$.

Kaufmann, F.-X. (1990). Zukunft der Familie. Stabilität, Stabilitätsrisiken und Wandel der familialen Lebensformen sowie ihre gesellschaftlichen und politischen Bedingungen. München: Beck.

Kaufmann, F.-X. (1994). Lässt sich Familie als gesellschaftliches Teilsystem begreifen? In: Herlth, A.//Brunner, E. J./Tyrell, H./Kriz, J. (Hrsg.). Abschied von der Normalfamilie? Partnerschaft kontra Elternschaft. Berlin: Springer, S. 42-63.

Kaufmann, F.-X. (2003). Varianten des Wohlfahrtstaats. Der deutsche Sozialstaat im internationalen Vergleich. Frankfurt/M.: Suhrkamp.

Kaufmann, J.-C. (1994). Schmutzige Wäsche. Zur ehelichen Konstruktion von Alltag. Konstanz: Universitätsverlag.

Kaufmann, J.-C. (1999). Mit Leib und Seele. Theorie der Haushaltstätigkeit. Konstanz: Universitätsverlag.

Keppler, A. (1994). Tischgespräche. Über Formen kommunikativer Vergemeinschaftung am Beispiel der Konversation in Familien. Frankfurt/M.: Suhrkamp.

Klein, T./Kopp, J. (Hrsg.) (1999). Scheidungsursachen aus soziologischer Sicht. Würzburg: Ergon.

Kohli, M. (1985). Die Institutionalisierung des Lebenslaufs. Historische Befunde und theoretische Argumente. Kölner Zeitschrift für Soziologie und Sozialpsychologie, 37, S. 1-29. 
Kohli, M./Szydlik, M. (Hrsg.) (2000). Generationen in Familie und Gesellschaft. Opladen: Leske + Budrich.

Koppetsch, C./Burkart, G. unter Mitarbeit von Maier, M. S. (1999). Die Illusion der Emanzipation. Zur Wirksamkeit latenter Geschlechtsnormen im Milieuvergleich. Konstanz: Universitätsverlag.

Kreyenfeld, M./Konietzka, D. (Hrsg.) (2006). Kinderlosigkeit in Deutschland. Wiesbaden: Sozialwissenschaftlicher Verlag VS.

Lauterbach, W. (2003). Mythenjägerin, oder was sonst? Zur Biografie der Familiensoziologie in den letzten 20 Jahren. In: Orth, B./Schwietring, T./Weiß, J. (Hrsg.). Soziologische Forschung. Stand und Perspektiven. Opladen: Leske + Budrich, S. 125-139.

Leira, A. (ed.) (1999). Family change. Practices, policies, and values (Comparative social research, Vol. 18). Stamford.: JAI, p. 1-29.

Leisering, L. (1992). Sozialstaat und demographischer Wandel. Wechselwirkungen, Generationenverhältnisse, politisch-institutionelle Steuerung. Frankfurt/M.: Campus.

Lenz, K. (2003). Familie - Abschied von einem Begriff. Erwägen - Wissen - Ethik, 14, S. 485-498.

Lenz, K. (2005). Familien als Ensemble persönlicher Beziehungen. In: Busch, F. W./NaveHerz, R.(Hrsg.). Familie und Gesellschaft. Beiträge zur Familienforschung. Oldenburg: BIS/Universität Oldenburg, S. 9-31.

Leu, H. R./Krappmann, L. (Hrsg.) (1999). Zwischen Autonomie und Verbundenheit. Bedingungen und Formen der Behauptung von Subjektivität. Frankfurt/M.: Suhrkamp.

Leupold, A. (1983). Liebe und Partnerschaft. Formen der Codierung von Ehen. Zeitschrift für Soziologie, 12, S. 297-327.

Luhmann, N. (1990). Sozialsystem Familie. In: Luhmann, N. Soziologische Aufklärung. Opladen: Westdeutscher Verlag, S. 196-217.

Lüscher, K. (1995). Was heißt heute Familie? Thesen zur Familienrhetorik. In: Gerhardt, U./Hradil, S./Lucke, D./Nauck, B. (Hrsg.) (1995). Familie der Zukunft. Lebensbedingungen und Lebensformen. Opladen: Leske + Budrich, S. 51-65.

Lüscher, K./Schultheis, F. (Hrsg.) (1993). Generationenbeziehungen in „postmodernen “ Gesellschaften. Konstanz: Universitätsverlag.

Lüscher, K./Schultheis, F./Wehrspaun, M. (Hrsg.) (1988). Die „postmoderne“ Familie. Familiale Strategien und Familienpolitik in einer Übergangszeit. Konstanz: Universitätsverlag.

Lüscher, K./Pillemer, K. (1998). Intergenerational ambivalence: a new approach to the study of parent-child relations in later life. Journal of Marriage and the Family, 60, p. 413-425.

Maihofer, A. (2002). Geschlecht und Sozialisation. Eine Problemskizze. Ethik und Sozialwissenschaften, 13, S. 13-26.

Matthias-Bleck, H. (2002). Soziologie der Lebensformen und der privaten Lebensführung. Soziale Welt, 53, S. 423-436.

Meyer, T. (1992). Modernisierung der Privatheit. Differenzierungs- und Individualisierungsprozesse des familialen Zusammenlebens. Opladen: Westdeutscher Verlag.

Mitterauer, M./Sieder, R. (1984). Vom Patriarchat zur Partnerschaft. München: Beck.

Nave-Herz, R. (1999). Diskontinuitäten zwischen Familie und Moderne. In: Friedrichs, J./Nave-Herz, R. Familiensoziologie. Oldenburg: Universität Oldenburg, S. 31-50.

Nave-Herz, R. (2004). Ehe- und Familiensoziologie. Eine Einführung in Geschichte, theoretische Ansätze und empirische Befunde. Weinheim: Juventa.

Osmond, M.W./Thorne, B. (1993). Feminist theories. The social construction of gender in families and societies. In: Boss, P. G. (Ed.). Sourcebook of family theory and methods: A contextual approach. New York: Plenum press, p. 591-623.

Ostner, I. (1997). Familie und Zivilgesellschaft. In: Schmals, K. M./Heinelt, H. (Hrsg.). Zivile Gesellschaft. Entwicklung - Defizite - Potentiale. Opladen: Leske + Budrich, S. 369-383. 
Parsons, T./Bales, R. F. (1955). Family, socialization, and the interaction process. Glencoe: Free Press.

Reckwitz, A. (2000). Die Transformation der Kulturtheorien. Zur Entwicklung eines Theorieprogramms. Weilerswist: Velbrück.

Schimank, U. (2002). Das zwiespältige Individuum. Zum Person-Gesellschaft-Arrangement der Moderne. Opladen: Leske + Budrich.

Schimank, U./Volkmann, U. (Hrsg.) (2000). Soziologische Gegenwartsdiagnosen. Opladen: Leske + Budrich/UTB.

Schmidt, U. (2002). Deutsche Familiensoziologie. Entwicklung nach dem Zweiten Weltkrieg. Wiesbaden: Westdeutscher Verlag.

Schneider, N. F./Matthias-Bleck, H. (Hrsg.) (2002). Elternschaft heute. Gesellschaftliche Rahmenbedingungen und individuelle Gestaltungsaufgaben. (Zeitschrift für Familienforschung, Sonderheft 2). Opladen: Leske + Budrich.

Schneider, N. F./Limmer, R./Ruckdeschel, K. (2002). Mobil, flexibel, gebunden. Familie und Beruf in der mobilen Gesellschaft. Frankfurt/M.: Campus.

Schneider, W. (2002). Von der familiensoziologischen Ordnung der Familie zu einer Soziologie des Privaten? Soziale Welt, 53, S. 375-396.

Schroer, M. (2000). Das Individuum der Gesellschaft. Frankfurt/M.: Suhrkamp.

Schulz, F./Blossfeld, H.-P. (2006). Wie verändert sich die häusliche Arbeitsteilung im Eheverlauf? Eine Längsschnittstudie der ersten 14 Ehejahre in Westdeutschland. Kölner Zeitschrift für Soziologie und Sozialpsychologie, 58, S. 23-49.

Scott, J./Treas, J./Richards, M. (eds.) (2004). The Blackwell companion to the sociology of families. Oxford: Blackwell.

Shorter, E. (1975). The making of the modern family. New York: Basic Books/London: Collins.

Sussmann, M. B./Steinmetz, S. K./Peterson, G. W. (Eds.) (1999). Handbook of marriage and the family. Second Edition. New York/London: Plenum.

Tölke, A./Hank, K. (Hrsg.) (2005). Männer - das ,,vernachlässigte“ Geschlecht in der Familienforschung. (Zeitschrift für Familienforschung, Sonderheft 4), Wiesbaden: VS-Verlag.

Turner, B. S. (1984). The body and society. Oxford: Blackwell.

Tyrell, H. (1986). Geschlechtliche Differenzierung und Geschlechterklassifikation. Kölner Zeitschrift für Soziologie und Sozialpsychologie, 38, S. 450-489.

Tyrell, H. (1988). Ehe und Familie. Institutionalisierung und Deinstitutionalisierung. In: Lüscher, K./Schultheis, F./Wehrspaun, M. (Hrsg.). Die „postmoderne“ Familie. Konstanz: Universitätsverlag, S. 145-156.

Tyrell, H. (2001). Das konflikttheoretische Defizit der Familiensoziologie. Überlegungen im Anschluss an Georg Simmel. In: Huinink, J./Strohmeier, K. P./Wagner, M. (Hrsg.). Solidarität in Partnerschaft und Familie. Zum Stand familiensoziologischer Theoriebildung. Würzburg: Ergon, S. 43-63.

Vaskovics, L. A. (2002). Pluralisierung der Elternrolle. Soziale, biologische, genetische und rechtliche Elternschaft.In: Brähler, E./Stöbel-Richter, Y./Hauffe, U. (Hrsg.). Vom Stammbaum zur Stammzelle. Reproduktionsmedizin, Pränataldiagnostik und menschlicher Rohstoff. Gießen: Psychosozial-Verlag, S. 29-43.

Vaskovics, L. A. (1995). Wiederentdeckung familialer Lebenswelten - ein Trend? (Einleitung zu:) Vaskovics, L. A./Garhammer, M. (Hrsg.). Familie - Soziologie familialer Lebenswelten (Soziologische Revue, Sonderband 3), München: Oldenbourg, S. 4-17.

Vaskovics, L. A./Garhammer, M. (1995). Editorial. In: Vaskovics, L. A./Garhammer, M. (Hrsg.). Familie - Soziologie familialer Lebenswelten (Soziologische Revue, Sonderband 3). München: Oldenbourg, S. 1-3. 
Wagner, M. (2001). Soziale Differenzierung, Gattenfamilie und Ehesolidarität. In: Huinink, J./Strohmeier, K. P./Wagner, M. (Hrsg.). Solidarität in Partnerschaft und Familie. Zum Stand familiensoziologischer Theoriebildung. Würzburg: Ergon, S. 19-42.

Wagner M./Schütze, Y. (Hrsg.) (1998). Verwandtschaft - Sozialwissenschaftliche Beiträge zu einem vernachlässigten Thema. Stuttgart: Enke.

Walby, S. (1997). Gender transformations. London: Routledge.

Willems, M. (1999). Vom „bloßen Menschen“ zum „einzigartigen Menschen“. Zur Entwicklung der Individualitätssemantik in Rationalismus, Empfindsamkeit und Sturm und Drang. In: Willems, H./Hahn, A. (Hrsg.). Identität und Moderne. Frankfurt/M.: Suhrkamp, S. 102-137.

Wimbauer, C. (2003). Geld und Liebe. Zur symbolischen Bedeutung von Geld in Paarbeziehungen. Frankfurt/M.: Campus.

Zelizer, V. A. (2005). The purchase of intimacy. Princeton: Princeton University Press.

Eingereicht am: 04.06.2006

Akzeptiert am: 03.07.2006

\author{
Anschrift des Autors: \\ Prof. Dr. Günter Burkart \\ Institut für Sozialwissenschaften \\ Universität Lüneburg \\ D-21332 Lüneburg
}

Email: burkart@uni-lueneburg.de 